\title{
A 3D distinct lattice spring model for elasticity and dynamic failure
}

\author{
Gao-Feng Zhao ${ }^{1}$, Jiannong Fang ${ }^{2}$ and Jian Zhao ${ }^{1, *, \dagger}$ \\ ${ }^{1}$ Ecole Polytechnique Federale de Lausanne (EPFL), Laboratory of Rock Mechanics, Station 18, \\ CH-1015 Lausanne, Switzerland \\ ${ }^{2}$ Ecole Polytechnique Federale de Lausanne (EPFL), Laboratory of Engineering and Environmental Geology, \\ Station 18, CH-1015 Lausanne, Switzerland
}

\begin{abstract}
SUMMARY
A 3D distinct lattice spring model (DLSM) is proposed where matter is discretized into individual particles linked by springs. The presented model is different from the conventional lattice spring models where a shear spring is introduced to model the multibody force by evaluating the spring deformation from the local strain rather than the particle displacement. By doing this, the proposed model can represent the diversity of Poisson's ratio without violating the rotational invariance. The local strain of the spring is calculated through a least square method which makes the model possessing meshless properties. Because of this and explicitly representing the microstructure, DLSM is able to model dynamic fracturing problems and can be used to study the microstructure influences. The material parameters inputted in the model is the conventional material parameters, e.g. the elastic modules and the Poisson's ratio. Relationships between microscopic spring parameters and macroscopic material constants are derived based on the Cauchy-Born rules and the hyperelastic theory. Numerical examples are presented to show the abilities and properties of DLSM in modeling elastic and dynamic failure problems. Copyright (C) 2010 John Wiley \& Sons, Ltd.
\end{abstract}

Received 22 September 2009; Revised 15 March 2010; Accepted 19 March 2010

KEY WORDS: 3D model; lattice spring model; microstructure; dynamic failure

\section{INTRODUCTION}

The classical elasticity theory could provide an adequate description of the macroscopic mechanical response of most materials, although they are actually heterogeneous when viewed at the microscopic level. However, dynamic fracturing of heterogeneous materials, such as rock and concrete, cannot be modeled realistically without appealing to their microstructures. This requires that a successful numerical method must be capable of considering not only the elastic stage, but also the formulation and evolution of micro discontinuities. Lattice models $[1,2]$ represent materials by a system of discrete units (e.g. particles) interacting via springs or, more generally, rheological elements. These discrete units are much coarser than the true atomic ones and may represent larger volumes of heterogeneities, such as grains or clusters of grains. Lattice models are close relative to the common finite element method (FEM) when dealing with elastic problems. Yet, due to their discrete nature, lattice models are known to be more suitable for complex fracturing simulation. For example, lattice models have been successfully applied to investigate the spatial cooperative effects of crack formation and heterogeneities in elastic-plastic [3] and elastic-brittle [4] systems.

\footnotetext{
${ }^{*}$ Correspondence to: Jian Zhao, Ecole Polytechnique Federale de Lausanne (EPFL), Laboratory of Rock Mechanics, Station 18, CH-1015 Lausanne, Switzerland.

†E-mail: jian.zhao@epfl.ch

Contract/grant sponsor: China Scholarship Council

Contract/grant sponsor: Swiss National Science Foundation; contract/grant number: 200021-116536 
However, for lattice models composed of normal springs transmitting central forces only, it is known that the modeled Poisson's ratio approaches in the limit of an infinite number of particles, a fixed value of e.g. 1/4 in three-dimensional cases. This kind of problem has been reported, for example, in the works of Beale and Strolovitz [5], Srolovitz and Beale [6], Nayfeh and Hefzy [7], and Donze and Magnier [8]. Such restriction is not suitable for many materials. It can be overcome by introducing non-central shear-type interactions between particles. One possible way is to add shear spring between each pair of particles. This approach was applied by Kawai [9] and Zubelewicz and Bazant [10]. It was investigated in greater detail by Griffiths and Mustoe [11] and refined by Cusatis et al. [12]. The addition of shear spring allows these lattice models to model Poisson's ratios less than 1/4. In addition to the particle displacements, these models also introduce the particle rotations as degrees of freedom, hence can be viewed as discretizations of micropolar continua. Another approach is to replace the normal springs by beams, which yields the so-called lattice beam models (LBMs) [13-16]. LBMs consider not only rotations but also bending deformations. There are arguments on the inclusion of the latter. For example, Cusatis et al. [12] pointed out that the bending of beams is not a characteristic of the physical phenomena in the microstructure. Attempts have also been made to tackle the problem without the cost of introducing rotational degrees of freedom. The models obtained in this way are usually called as lattice spring models (LSMs). Hassold and Srolovitz [17] proposed a method to modify the Poisson's ratio by introducing a harmonic potential for rotation of bonds from their initial orientation. Here bonds denote the connecting elements between particles. A non-central two-body interaction limiting the rotational freedom of bonds is introduced in the Born spring model $[18,19]$ to allow a broad choice of Poisson's ratio. Nevertheless, rotational invariance of the models can only be recovered if a threebody interaction is considered. The Kirkwood-Keating spring model [20,21] introduces angular springs to penalize the angular variations between the contiguous bonds incident onto the same node. Modeling the multibody interactions by angular springs is not so convenient (the angular terms are nonlinear functions of displacements) and may bring difficulties in the failure modeling. Although the aforementioned approaches relax the restriction, they cannot model Poisson's ratios greater than $1 / 4$.

In this paper we propose an alternative 3D dynamic lattice spring model which overcomes the restriction on Poisson's ratio while preserving the rotational invariance. The model includes a normal spring and a multibody shear-type spring for each pair of lattice points (particles). The lattice structure can be either random or regular. It shall be shown that negative shear stiffness can be adopted in the proposed model to allow the full range of Poisson's ratio of elastic solid to be modeled. The deformation of the shear springs is evaluated by using the local strain rather than the particle displacement. It shall be proven that this technique makes the model rotationally invariant. The local strain is calculated by a fully meshless approach which avoids meshing or re-meshing in case of fracture simulation. The method of solving system equations is the same as used in distinct element method (DEM) developed by Cundall and Strack [22]. In view of the multibody shear spring and the solver used in the model, we name it as distinct lattice spring model (DLSM). In DLSM, there is no need to form the global stiffness matrix and only a local interaction is considered during calculation. This is very suitable for large-scale parallel computing implementation. The context of the paper is organized as following. First, the proposed model and associated numerical techniques are described. Second, the relationship between micro spring stiffness and macro elastic constants is derived. Then, the model is validated through numerical simulation of three elastic problems, one wave propagation problem and two dynamic failure problems. The paper ends with some conclusions and remarks.

\section{THE DLSM}

This section will give the basic conception, formulations, and numerical techniques used in the DLSM. At the beginning, the physical model and equation of motion of the system and the solution method will be introduced. Then, mathematical formulation of the interactions between particles 

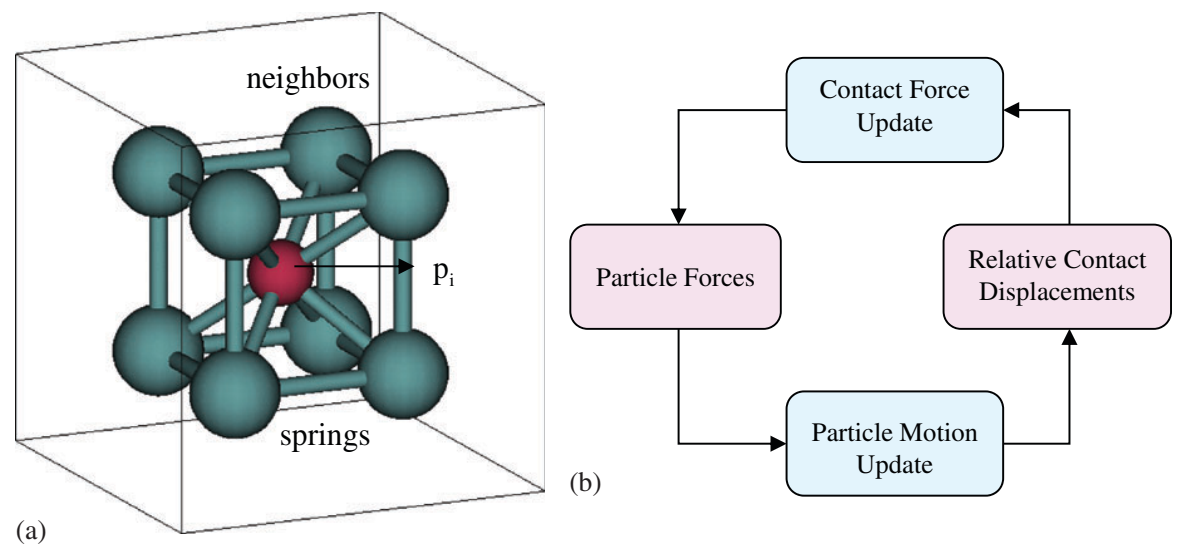

Figure 1. The physical model and the calculation cycle of DLSM: (a) the physical model of DLSM and (b) calculation cycle.

will be addressed. The multibody shear spring is introduced in a distinct way using the local strain to evaluate the shear deformation. A least square method is used to obtain the local strain. The damping scheme and time step selection will be discussed in the last part.

\subsection{Physical model and system equations}

In DLSM, material is discretized into mass particles with different sizes. Whenever the gap between two particles is smaller than a given threshold value, the two particles are linked together through a bond between their center points (as shown in Figure 1(a)), which consists of normal and shear springs. The threshold value will influence the lattice structure of the model; different threshold values would produce different lattice structures. This will be discussed later. The particles and bonds form a network system representing the material. For this system, its equation of motion can be expressed as

$$
[\mathbf{K}] \mathbf{u}+[\mathbf{C}] \dot{\mathbf{u}}+[\mathbf{M}] \ddot{\mathbf{u}}=\mathbf{F}(t)
$$

where $\mathbf{u}$ represent the vector of particle displacement, $[\mathbf{K}]$ the stiffness matrix, $[\mathbf{M}]$ the diagonal mass matrix, $[\mathbf{C}]$ the damping matrix, and $\mathbf{F}(t)$ the vector of external force. Equation (1) is solved by using the explicit central finite difference scheme, which was reported by Rougier et al. [23] as the most efficient and robust method among the various explicit integration schemes. The calculation cycle is illustrated in Figure 1(b). Given the particle displacements (either prescribed initially or obtained from the previous time step), new contacts and broken bonds are detected. The list of neighboring particles for each particle is updated. Then, contact and spring forces between particles are calculated according to the prescribed force-displacement relations. The particle velocity is advanced individually as

$$
\dot{\mathbf{u}}_{i}^{(t+\Delta t / 2)}=\dot{\mathbf{u}}_{i}^{(t-\Delta t / 2)}+\frac{\sum \mathbf{F}_{j}^{(t)}}{m_{\mathrm{p}}} \Delta t
$$

where $\dot{\mathbf{u}}_{i}^{(t+\Delta t / 2)}$ is the particle velocity at $t+\Delta t / 2, \dot{\mathbf{u}}_{i}^{(t-\Delta t / 2)}$ the particle velocity at $t-\Delta t / 2, m_{\mathrm{p}}$ the particle mass, $\sum \mathbf{F}_{j}^{(t)}$ the sum of forces acting on the particle $i$ including applied external forces, and $\Delta t$ the time step. Finally, the new displacement of particle is obtained as

$$
\mathbf{u}_{i}^{(t+\Delta t)}=\mathbf{u}_{i}^{(t)}+\dot{\mathbf{u}}_{i}^{(t+\Delta t / 2)} \Delta t
$$

where $\mathbf{u}_{i}^{(t+\Delta t)}$ is the displacement at $t+\Delta t$ and $\mathbf{u}_{i}^{(t)}$ the displacement at $t$. This central difference scheme is equivalent to the Newton's second law used in DEM and molecular dynamics (MD) 

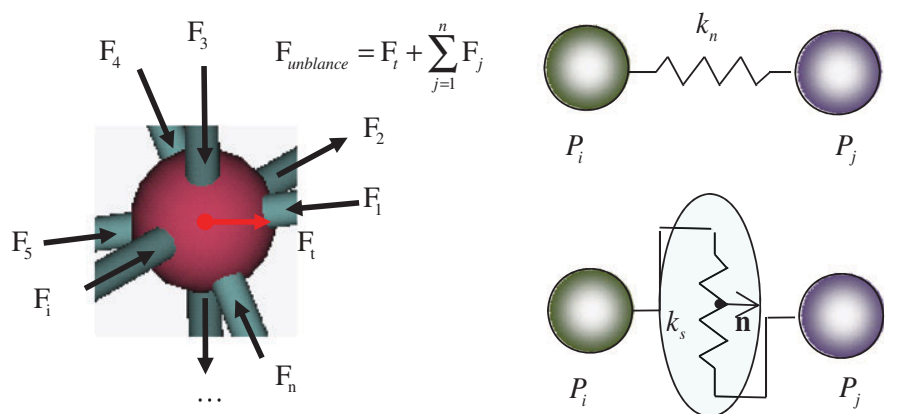

(a)

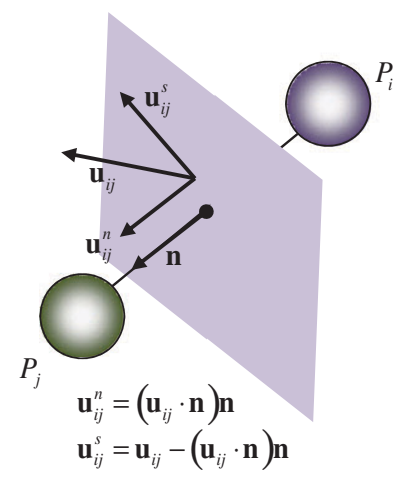

(c) (b)
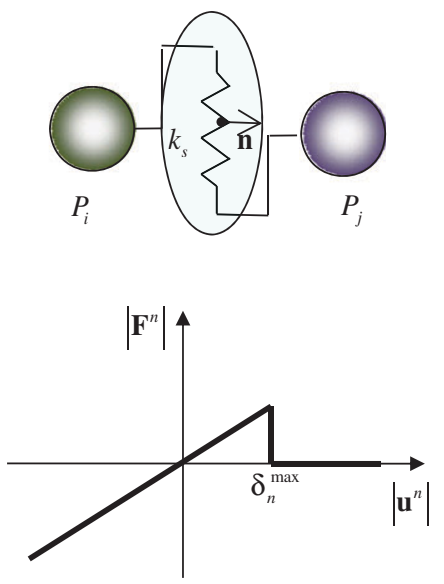

(d)

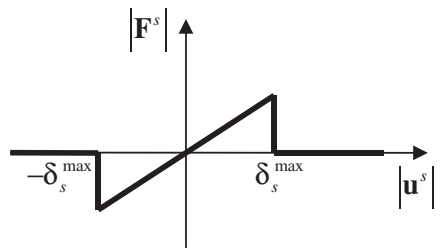

Figure 2. The force and displacement relationships between two particles and the micro constitutive laws: (a) the forces on one particle; (b) the normal and shear springs; (c) the shear and normal displacement; and (d) constitutive laws of lattice springs.

simulations. In the next subsection, the formulation of the interaction forces between particles is described.

\subsection{The interactions between particles}

Figure 2(a) shows the forces exerted on one particle. These forces are made up of the external force and contact force between particles. The interaction between linked particles is represented by one normal spring and one shear spring as illustrated in Figure 2(b). Different from the conventional LSMs, the shear spring is introduced to model the multibody non-central interaction and make the model capable of handling problems with a variable choice of Poisson's ratio. The normal spring is implemented in a conventional way. For a bond connecting particle $i$ and particle $j$, the normal unit vector $\mathbf{n}=\left(n_{x}, n_{y}, n_{z}\right)^{\mathrm{T}}$ pointing from particle $i$ to particle $j$ is defined (see Figure 2(c)). The relative displacement is calculated as

$$
\mathbf{u}_{i j}=\mathbf{u}_{j}-\mathbf{u}_{i}
$$

The normal force between the two particles is defined as

$$
\mathbf{F}_{i j}^{\mathrm{n}}=k_{\mathrm{n}} \mathbf{u}_{i j}^{\mathrm{n}}
$$

where $k_{\mathrm{n}}$ is the stiffness of the normal spring and $\mathbf{u}_{i j}^{\mathrm{n}}=\left(\mathbf{u}_{i j} \bullet \mathbf{n}\right) \mathbf{n}$ is the vector of normal displacement (see Figure 2(c)).

For the shear spring, the relative shear displacement between two particles can be obtained simply as $\mathbf{u}_{i j}^{\mathrm{s}}=\mathbf{u}_{i j}-\mathbf{u}_{i j}^{\mathrm{n}}$ like in some conventional LSMs. However, it is straightforward to show that the shearing force calculated in this way is not rotationally invariant. To overcome the problem, we propose a local strain-based method. Assuming the strain at the two particles is evaluated as 
$[\varepsilon]_{i}$ and $[\varepsilon]_{j}$, respectively, the strain state of the connecting bond is given as the average of the two particle strains:

$$
[\varepsilon]_{\text {bond }}=\frac{[\varepsilon]_{i}+[\varepsilon]_{j}}{2}
$$

where

$$
[\varepsilon]=\left[\begin{array}{ccc}
\varepsilon_{x x} & \varepsilon_{x y} & \varepsilon_{x z} \\
\varepsilon_{y x} & \varepsilon_{y y} & \varepsilon_{y z} \\
\varepsilon_{z x} & \varepsilon_{z y} & \varepsilon_{z z}
\end{array}\right] .
$$

The shear displacement vector is obtained as

$$
\hat{\mathbf{u}}_{i j}^{\mathrm{s}}=[\varepsilon]_{\mathrm{bond}} \cdot \mathbf{n} l-\left(\left([\varepsilon]_{\mathrm{bond}} \cdot \mathbf{n} l\right) \cdot \mathbf{n}\right) \mathbf{n}
$$

where $l$ is the initial bond length, i.e. the initial distance between the pair of particles. Then the shearing force between the two particles reads as

$$
\mathbf{F}_{i j}^{\mathrm{s}}=k_{\mathrm{s}} \hat{\mathbf{u}}_{i j}^{\mathrm{s}}
$$

where $k_{\mathrm{S}}$ is the stiffness of the shear spring. The proposed method here together with the strain calculation procedure described in the next subsection ensures that the model preserves the rotational invariance of LSM consisting of normal springs only. A proof of this is given in Appendix.

Equations (5) and (8) are valid for unbroken bonds. The failure criterion used in DLSM is shown in Figure 2(d). When the normal or shear displacement of the bond exceeds the prescribed value, the bond is broken and becomes a contact bond for which only a normal spring with zero strength is applied. At the current stage, only a simple fracture criterion is adopted and a more comprehensive study on the fracture criteria is needed. The proposed model has only two spring parameters and two failure parameters. Hence, it is suitable for microscopic modeling as the lesser input parameters the easier it is to observe and study the microstructure influence on the mechanical response of materials.

\subsection{The least square method for obtaining the local strain}

In DLSM, the local strain of one particle is evaluated by a least square scheme which only uses the displacement of itself and other particles which have intact bonds with the particle. By doing so, discontinuities (e.g. fracture/crack) could be directly considered without using the 'visibility criterion' adopted by most meshless methods. First, assume the displacement function within a small volume (cloud) around the particle can be approximated as a linear function

$$
f(x, y, z)=a x+b y+c z+d
$$

Given the displacement of all the particles in the cloud, the coefficients of Equation (9) can be estimated by using the least square method. Taking the $x$-component of $\boldsymbol{u}$ as an example, it is achieved by minimizing the quadratic equation

$$
J=\sum_{j=1}^{n}\left(u_{j x}-\hat{u}_{j x}\right)^{2}
$$

where $n$ is the number of particles in the cloud and $\hat{u}_{j x}$ is the approximated $x$-component of $\boldsymbol{u}$ at particle $j$ which is given as

$$
\hat{u}_{j x}=a x_{j}+b y_{j}+c z_{j}+d
$$

The coefficients are obtained as

$$
\alpha=\left(\begin{array}{llll}
a & b & c & d
\end{array}\right)^{\mathrm{T}}=\left(\mathbf{A}^{\mathrm{T}} \mathbf{A}\right)^{-1}\left(\mathbf{A}^{\mathrm{T}} \boldsymbol{\beta}\right)
$$


where

$$
\mathbf{A}=\left[\begin{array}{cccc}
x_{1} & y_{1} & z_{1} & 1 \\
x_{2} & y_{2} & z_{2} & 1 \\
\vdots & \vdots & \vdots & \vdots \\
x_{n} & y_{n} & z_{n} & 1
\end{array}\right] \text { and } \boldsymbol{\beta}=\left[\begin{array}{c}
u_{1 x} \\
u_{2 x} \\
\vdots \\
u_{n x}
\end{array}\right]
$$

Using this approach, the approximated displacement field in the cloud is obtained as linear functions, of which first-order derivatives yield the strain, e.g. $\varepsilon_{x x}=\partial u_{x} / \partial x=a$.

The least square method used in DLSM makes the model fully meshless and the inverse matrix of $A^{\mathrm{T}} A(4 \times 4)$ can be calculated very fast. In a practical simulation, the inverse of $A^{\mathrm{T}} A$ may not exist in some conditions. In this case shear spring will not be considered anymore for the relevant particles. Since the least square approximation is first-order consistent, it can be proven that the calculated strain is independent of rotational displacement (see Appendix).

\subsection{Damping and time step}

The solution scheme used in DLSM is conditionally stable. To keep the computation stable, the time step could be chosen according to the requirement that it is less than the time needed for elastic wave propagation through the smallest element of the model. This leads to

$$
\Delta t_{\mathbf{n}}=\min \left(\frac{l_{i}}{C_{\mathrm{p}}}\right)
$$

where $C_{\mathrm{p}}$ is the P-wave velocity of the model and $l_{i}$ is the $i$ th spring length of the model. The P-wave velocity of the model can be obtained through

$$
C_{\mathrm{p}}=\sqrt{\frac{K+4 G / 3}{\rho}}
$$

where $\rho$ is the density, $K$ and $G$ are the bulk and shear elastic modules of the model which have a relationship with the elastic module $E$ and Poisson's ratio $u$ as follows:

$$
\begin{aligned}
& K=\frac{E}{3(1-2 u)} \\
& G=\frac{E}{2(1+u)}
\end{aligned}
$$

It should be mentioned that the input parameters of DLSM are macroscopic elastic parameters rather than microscopic spring parameters. This makes the DLSM modeling consistent with the conventional FEM modeling. The relationship between macroscopic elastic parameters and microscopic spring parameters will be introduced in the next section.

Mechanical damping is used in DLSM to obtain static solutions. For static analysis, the approach is conceptually similar to dynamic relaxation proposed by Otter et al. [24]. The equations of motion are damped to reach a force equilibrium state as quickly as possible under the applied initial and boundary conditions. A local damping scheme, which is used in the DEM to overcome the difficulties of the velocity-proportional damping, is adopted in DLSM. When the local damping is incorporated, the equation of motion, Equation (2), is replaced by the following equation:

$$
\dot{\mathbf{u}}_{i}^{(t+\Delta t / 2)}=\dot{\mathbf{u}}_{i}^{(t-\Delta t / 2)}+\left\{\sum \mathbf{F}_{i}^{(t)}-\alpha\left|\sum \mathbf{F}_{i}^{(t)}\right| \operatorname{sgn}\left(\dot{\mathbf{u}}_{i}^{(t-\Delta t / 2)}\right)\right\} \frac{\Delta t}{m_{\mathrm{p}}}
$$

where $\alpha$ is the damping constant (set to 0.8 in DLSM) which is dimensionless and independent of mechanical properties and boundary conditions. This type of damping is equivalent to a local form 
of adaptive damping. By using this damping scheme, the damping forces vanish for steady-state conditions. The local damping is reported to be under-damped in general. For the dynamic case, DLSM switches off the damping term $(\alpha=0)$ and neglects the strain rate effect. The strain rate effect is still a debatable issue, for example Li et al. [25] believe that the strain rate is just caused by the effect of inertia.

\section{RELATIONSHIP BETWEEN SPRING PARAMETERS AND ELASTIC CONSTANTS}

In this section, the relationship between spring parameters and elastic constants is derived by following the approach used in the virtual multidimensional internal bond model [26, 27]. The total strain energy stored per unit volume is

$$
\begin{aligned}
\Pi & =\frac{\sum \Pi_{\mathrm{b}}}{V}=\frac{\sum\left(k_{\mathrm{n}} \mathbf{u}_{i j}^{\mathrm{n}} \cdot \mathbf{u}_{i j}^{\mathrm{n}}+k_{\mathrm{s}} \hat{\mathbf{u}}_{i j}^{\mathrm{s}} \cdot \hat{\mathbf{u}}_{i j}^{\mathrm{s}}\right)}{2 V} \\
& =\sum \frac{l^{2}\left(k_{\mathrm{n}} \xi_{i} \varepsilon_{i j} \xi_{j} \xi_{n} \varepsilon_{n m} \xi_{m}+k_{\mathrm{s}}\left(\varepsilon_{k l} \xi_{l}-\xi_{i} \varepsilon_{i j} \xi_{j} \xi_{k}\right)\left(\varepsilon_{k m} \xi_{m}-\xi_{n} \varepsilon_{n m} \xi_{m} \xi_{k}\right)\right)}{2 V}
\end{aligned}
$$

where $V$ is the volume of the modeling domain and $\xi_{i}$ denotes the $i$-th component of the normal vector $\mathbf{n}$. The stress tensor of the continuum can be obtained through the Cauchy-Born rule [28] and the hyperelastic theory [29], and it can be written as

$$
\sigma_{i j}=\frac{\partial \Pi}{\partial \varepsilon_{i j}}=\sum \frac{l^{2}\left(k_{\mathrm{n}} \xi_{i} \xi_{j} \xi_{n} \varepsilon_{n m} \xi_{m}+k_{\mathrm{s}}\left(\varepsilon_{i k} \xi_{k} \xi_{j}-\xi_{n} \varepsilon_{n m} \xi_{m} \xi_{i} \xi_{j}\right)\right)}{V}
$$

The elastic modulus is expressed as

$$
c_{i j n m}=\frac{\partial^{2} \Pi}{\partial \varepsilon_{i j} \partial \varepsilon_{n m}}=\sum \frac{l^{2}\left(k_{\mathrm{n}} \xi_{i} \xi_{j} \xi_{n} \xi_{m}+k_{\mathrm{s}}\left(\delta_{i n} \xi_{j} \xi_{m}-\xi_{i} \xi_{j} \xi_{n} \xi_{m}\right)\right)}{V}
$$

When the total number of bonds is large enough, Equation (21) can be written in the integral form as

$$
c_{i j n m}=\frac{1}{V} \int_{l_{1}}^{l_{2}} \int_{0}^{\pi} \int_{0}^{2 \pi} l^{2}\left(k_{\mathrm{n}} \xi_{i} \xi_{j} \xi_{n} \xi_{m}+k_{\mathrm{s}}\left(\delta_{i n} \xi_{j} \xi_{m}-\xi_{i} \xi_{j} \xi_{n} \xi_{m}\right)\right) D(l, \theta, \phi) \sin (\theta) \mathrm{d} \theta \mathrm{d} \phi \mathrm{d} l
$$

where $D(l, \theta, \phi) \sin (\theta) \mathrm{d} \theta \mathrm{d} \phi \mathrm{d} l$ is the number of multidimensional internal bonds in the undeformed solid with bond length between $(l, l+\mathrm{d} l)$ and bond orientation between $(\theta, \theta+\mathrm{d} \theta)$ and $(\phi, \phi+\mathrm{d} \phi)$. For the isotropic material, the bonds distribute uniformly in each direction. So the bond distribution function $D(l, \theta, \phi)$ is reduced to $N(l) / 2 \pi$ with $N(l) \mathrm{d} l$ being the number of multidimensional internal bonds with length between $(l, l+\mathrm{d} l)$. In numerical methods e.g. FEM, the elastic tensor $c_{i j n m}$ is often written in the elastic matrix form as follows:

$$
\mathbf{\Omega}=\left[\begin{array}{llllll}
C_{1111} & C_{1122} & C_{1133} & \frac{1}{2}\left(C_{1112}+C_{1121}\right) & \frac{1}{2}\left(C_{1132}+C_{1123}\right) & \frac{1}{2}\left(C_{1113}+C_{1131}\right) \\
C_{2211} & C_{2222} & C_{2233} & \frac{1}{2}\left(C_{2212}+C_{2221}\right) & \frac{1}{2}\left(C_{2232}+C_{2223}\right) & \frac{1}{2}\left(C_{2213}+C_{2231}\right) \\
C_{3311} & C_{3322} & C_{3333} & \frac{1}{2}\left(C_{3312}+C_{3321}\right) & \frac{1}{2}\left(C_{3332}+C_{3323}\right) & \frac{1}{2}\left(C_{3313}+C_{3331}\right) \\
C_{1211} & C_{1222} & C_{1233} & \frac{1}{2}\left(C_{1212}+C_{1221}\right) & \frac{1}{2}\left(C_{1232}+C_{1223}\right) & \frac{1}{2}\left(C_{1213}+C_{1231}\right) \\
C_{2311} & C_{2322} & C_{2333} & \frac{1}{2}\left(C_{2312}+C_{2321}\right) & \frac{1}{2}\left(C_{2332}+C_{2323}\right) & \frac{1}{2}\left(C_{2313}+C_{2331}\right) \\
C_{1311} & C_{1322} & C_{1333} & \frac{1}{2}\left(C_{1312}+C_{1321}\right) & \frac{1}{2}\left(C_{1332}+C_{1323}\right) & \frac{1}{2}\left(C_{1313}+C_{1331}\right)
\end{array}\right]
$$


For the linear elastic cases, the tangent modulus is equal to the secant modulus and Equation (22) can be considered as the secant modulus. So the following relationship exists:

$$
\boldsymbol{\sigma}=\boldsymbol{\Omega} \cdot \varepsilon
$$

where $\boldsymbol{\sigma}=\left[\sigma_{11}, \sigma_{22}, \sigma_{33}, \sigma_{12}, \sigma_{23}, \sigma_{13}\right]^{\mathrm{T}}$ and $\varepsilon=\left[\varepsilon_{11}, \varepsilon_{22}, \varepsilon_{33}, 2 \varepsilon_{12}, 2 \varepsilon_{23}, 2 \varepsilon_{13}\right]^{\mathrm{T}}$. Here $\sigma_{i j}$ and $\varepsilon_{i j}$ are the components of stress and strain tensors, respectively. By integrating Equation (22) for the case of isotropic material and using Equation (23), the corresponding elastic matrix is obtained as:

$$
\boldsymbol{\Omega}=\frac{\int_{l_{1}}^{l_{2}} l^{2} N(l) \mathrm{d} l}{15 L^{3}}\left[\begin{array}{cccccc}
3 k_{\mathrm{n}}+2 k_{\mathrm{s}} & k_{\mathrm{n}}-k_{\mathrm{s}} & k_{\mathrm{n}}-k_{\mathrm{s}} & 0 & 0 & 0 \\
& 3 k_{\mathrm{n}}+2 k_{\mathrm{s}} & k_{\mathrm{n}}-k_{\mathrm{s}} & 0 & 0 & 0 \\
& & 3 k_{\mathrm{n}}+2 k_{\mathrm{s}} & 0 & 0 & 0 \\
& & & k_{\mathrm{n}}+1.5 k_{\mathrm{s}} & 0 & 0 \\
& & & & k_{\mathrm{n}}+1.5 k_{\mathrm{s}} & 0 \\
& & & & & k_{\mathrm{n}}+1.5 k_{\mathrm{s}}
\end{array}\right]
$$

Let $\alpha^{3 \mathrm{D}}=\int_{l_{1}}^{l_{2}} l^{2} N(l) \mathrm{d} l / L^{3}$, then the relationship between the micromechanical parameters $k_{\mathrm{n}}, k_{\mathrm{s}}$ and the macro material constants, i.e. the Young's modulus $E$ and the Poisson ratio $v$ can be obtained by comparing Equation (25) to the classical elastic matrix as follows:

$$
\begin{aligned}
& k_{\mathrm{n}}=\frac{3 E}{\alpha^{3 \mathrm{D}}(1-2 v)} \\
& k_{\mathrm{s}}=\frac{3(1-4 v) E}{\alpha^{3 \mathrm{D}}(1+v)(1-2 v)}
\end{aligned}
$$

Here $\alpha^{3 \mathrm{D}}$ can be regarded as a microstructure geometry coefficient.

Given the geometry data of the LSM, $\alpha^{3 \mathrm{D}}$ can be estimated through

$$
\alpha^{3 \mathrm{D}}=\frac{\sum l_{i}^{2}}{V}
$$

where $l_{i}$ is the original length of the $i$-th bond. Equations (26) and (27) are used to estimate $k_{\mathrm{n}}$ and $k_{\mathrm{s}}$ of the proposed DLSM for numerical simulation of elastic problems. In the next section, numerical examples of validation and application of the model is presented.

\section{NUMERICAL EXAMPLES}

\subsection{Simple cube under pure tensile loading}

In this section the pure tensile loading of a cubic cell with a length of $10 \mathrm{~mm}$ is simulated. The purpose is to study the influence of lattice structure on the mechanical response. The model setup and three different particle distributions are shown in Figure 3(a). The first one is the simple cubic model for which particle arrangement is shown in Figure 3(b). The simple cubic structure is one of the most common crystal structures. The second one is the body-centered cubic (BCC) model. The third one is the random distributed model which is generated by the PFC3D code of Itasca Consulting group and the details of this generation method were described in [30]. The lattice structure is formed according to the threshold value of particle gap. Different threshold values would lead to different lattice structures. Figure 4 shows the structures of the three particle models. For the simple cubic case, there are three types of structure: cubic I (shown in Figure 4(a)), cubic II (shown in Figure 4(b)), and cubic III (shown in Figure 4(c)). For the BCC model, two types of structure are considered, the first one is BCC I (shown in Figure 4(d)) and the second is BCC II (shown in Figure 4(e)). For the random structure, only 


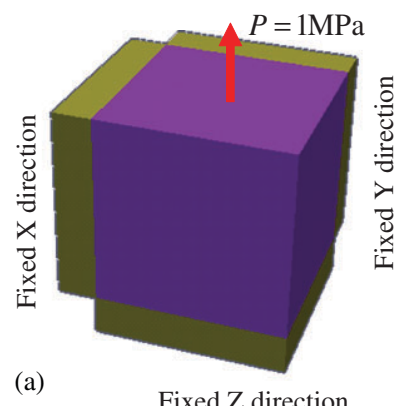

(b)
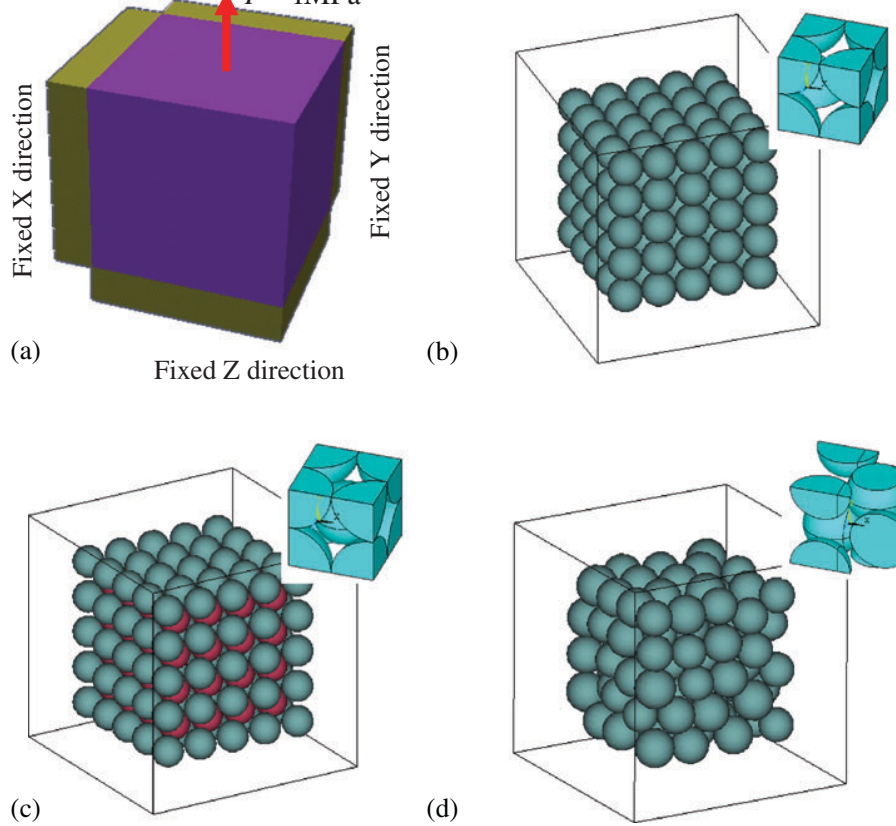

(d)

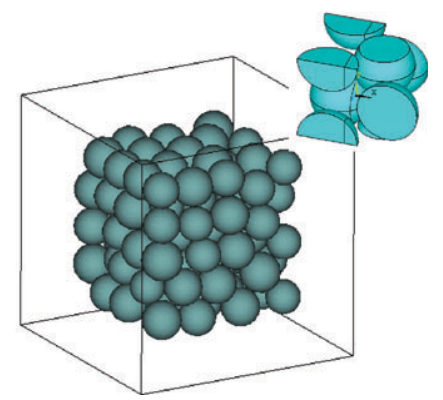

Figure 3. The $1 / 8$ part of the cubic cell under uniaxial tensile loading and different microstructures: (a) 1/8 part of the specimen; (b) simple cubic structure; (c) body centered structure; and (d) random structure.
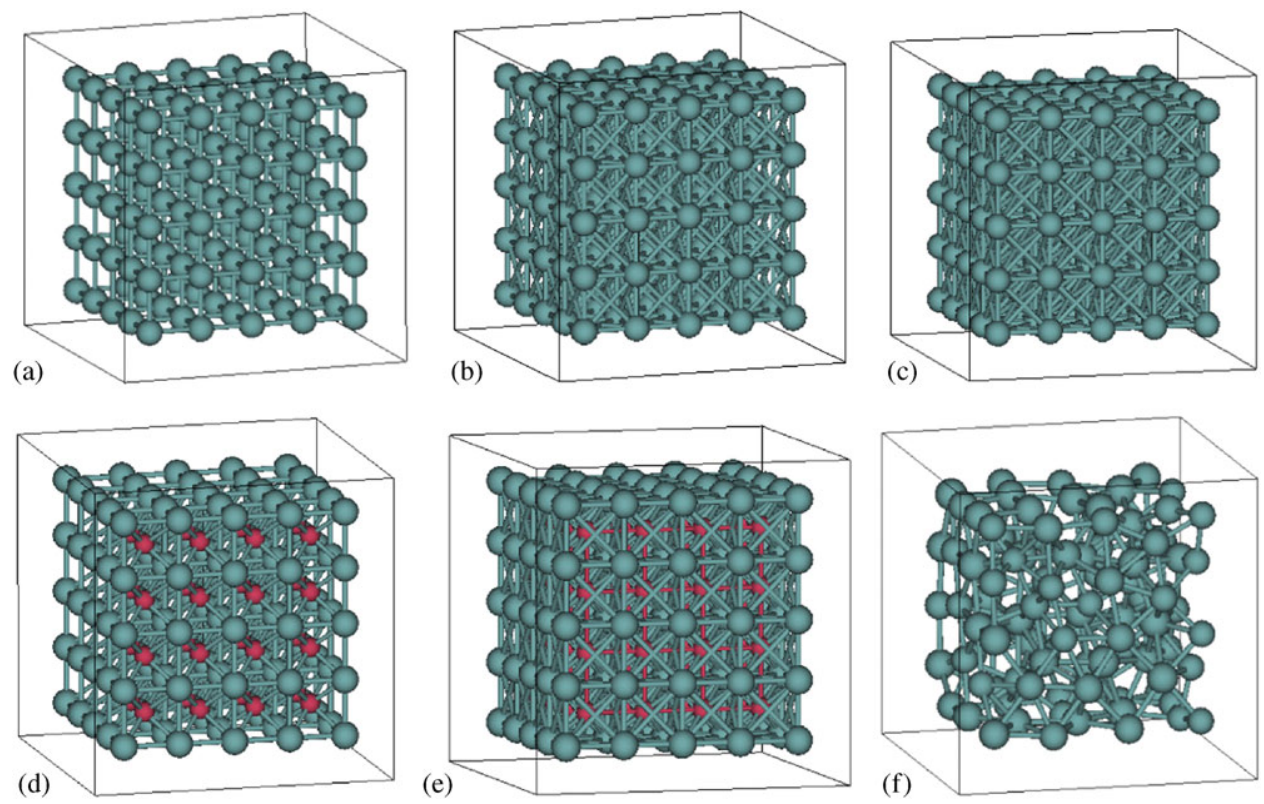

Figure 4. Different lattice structures created by using different interaction ranges: (a) Cubic I; (b) Cubic II; (c) Cubic III; (d) BCC I; (e) BCC II; and (f) random structure.

one case is studied (see Figure 4(f)). The number of particles is 125 in the simple cubic model, 189 in the BCC model and 100 in the random model. Simulations are performed to study the microstructure influence on the mechanical response of the lattice model. All the models are calculated for different Poisson's ratios ranging from 0.2 to 0.3 and a fixed elastic modulus $10 \mathrm{GPa}$. The time step is selected according to Equation (14). The simulation results show that this 

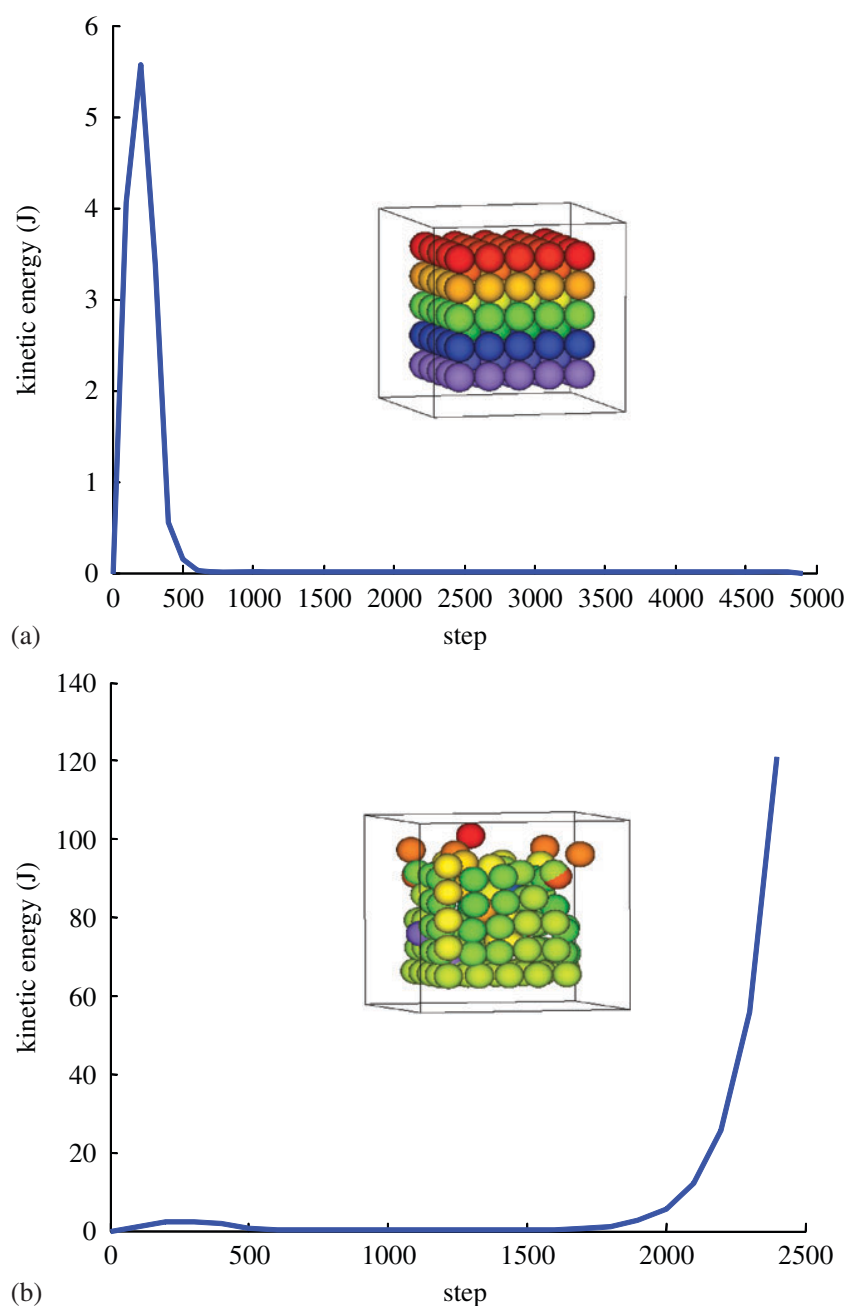

Figure 5. The stable (a) and unstable (b) case of DLSM for simulating tensile loading of a simple cube.

time step selection criterion is correct. For obtaining a static solution, the local damping scheme is used.

Equation (26) indicates that a shear spring of negative stiffness would occur when the Poisson's ratio is greater than $1 / 4$. The negative spring seems non-physical, but for DLSM, shear spring is introduced in a way to model the multibody interactions, so negative stiffness may result from a structural effect of the high Poisson's ratio material. In reality, materials with negative stiffness are also reported and used for extreme damping in composite materials [31]. From our simulations, it is shown that the lattice model is convergent for most of the lattice structures except for the cubic I case. Figure 5 shows the kinetic energy varying with the iteration steps during the calculation using the cubic I model. For the stable case $(v \leqslant 1 / 4)$, the model will reach a static equilibrium state and the kinetic energy of the model will be zero as shown in Figure 5(a). Whereas for the unstable case the kinetic energy increases to a divergent state and the model collapses as shown in Figure 5(b). Table I lists the simulation results of different models with different Poisson's ratios. Results shown are the $z$-direction displacement of the center particle in the top surface. As the particle is a sphere with a radius of $1 \mathrm{~mm}$ and the boundary force $(P=1 \mathrm{MPa})$ is applied at the center of the sphere, the effective length is $H_{\mathrm{e}}=8 \mathrm{~mm}$ (the sample length minus twice the radius). So the expected value for the $z$-direction displacement is $H_{\mathrm{e}} P / E=0.0008 \mathrm{~mm}$ for all the cases. From the results we can see that the cubic I and cubic III are not good for the simulation of isotropic elasticity. Because the first one is unstable for Poisson's ratio greater than $1 / 4$ and the 
Table I. The $z$-direction displacement predicted by different microstructure models with different Poisson's ratios for the simple cube problem.

\begin{tabular}{lccr}
\hline & \multicolumn{3}{c}{ Poisson's ratio } \\
\cline { 2 - 4 }$u_{z}^{*}(\mathrm{~mm})$ & 0.2 & 0.25 & 0.30 \\
\hline Models & & & \\
Cubic I & 0.00038 & 0.00032 & Unstable \\
Cubic II & 0.00058 & 0.00058 & 0.00057 \\
Cubic III & 0.00072 & 0.00086 & 0.00120 \\
BCC I & 0.00057 & 0.00058 & 0.00058 \\
BCC II & 0.00060 & 0.00062 & 0.00065 \\
Rand & 0.00070 & 0.00073 & 0.00078 \\
\hline
\end{tabular}

${ }^{*}$ The expected value is $0.0008 \mathrm{~mm}$ for all the cases.

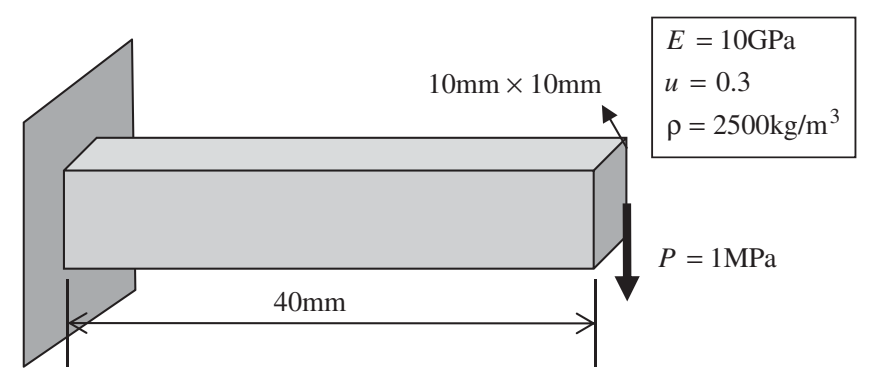

Figure 6. The boundary conditions and material parameters for the beam bending problem.

second one cannot reflect well the correct Poisson's effect (the displacement is too sensitive to the change of Poisson's ratio). We see that the predicted displacements of the measured particle have relative errors of about $20 \%$ for each case. This is due to a small number of particles being used. Our purpose in this section is not to compare the elasticity solution with the lattice model but to study the structure influence on the mechanical behavior of the proposed lattice model. In the following section a more complex problem will be simulated to verify the ability of DLSM for modeling the elasticity.

\subsection{Beam subjected to bending}

The previous example shows that the lattice structure has great influence on the simulation results. It is found that the Cubic I lattice structure is not stable and cannot be used to model the elasticity correctly. In this section, a problem with more complex loading conditions is simulated using a large number of particles to further check whether DLSM could reproduce the elasticity correctly. Another purpose of this example is to screen out the most proper lattice structures. Figure 6 gives the geometry information, the boundary conditions, and the material parameters. The left end of the beam is fixed and the right end is subjected to a shearing force of $1 \mathrm{MPa}$. The beam will undergo a complex stress condition, i.e. tensile, compressive, and shear stress would appear. Figure 7(a) shows the FEM model and the DLSM model of different structures. The FEM solution is used as the reference one for comparing with the DLSM results. The resolution of the FEM model is $10 \times 10 \times 40$ using 4000 evenly distributed eight-node elements. The lattice model has 4000 particles in case of regular structure and 4965 particles in case of random structure. Figure 7 shows the simulation results of FEM and DLSM. It is found that the lattice model could reproduce the same displacement distribution as the FEM model. This means the lattice model can be regarded as a valid representation of isotropic elastic material. Quantitative comparison is given in Figure 8, where the $y$-direction displacements of the middle line of the beam predicted by FEM and DLSM are shown. The results of the lattice model with cubic II, BCC I, BCC II, and random structure have a good agreement with the FEM solution. However, the model with cubic III structure does not yield satisfactory results. This implies that the cubic III structure 


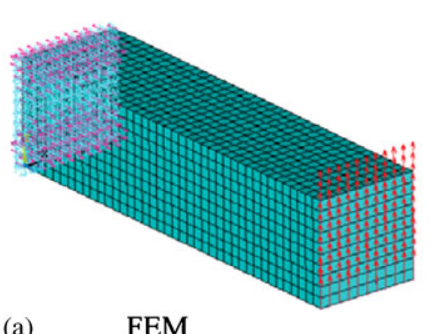

(a)

FEM

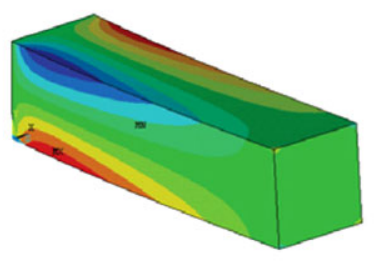

(b)

FEM
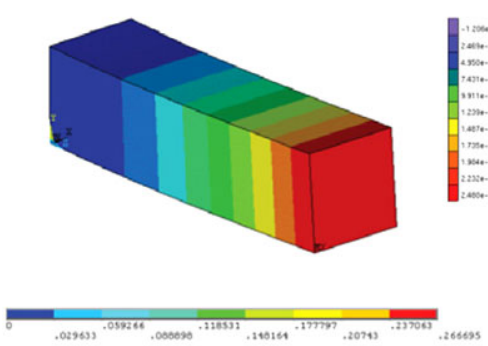

(c)

FEM

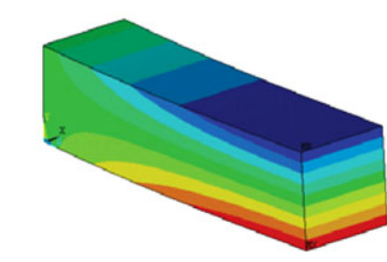

(d)

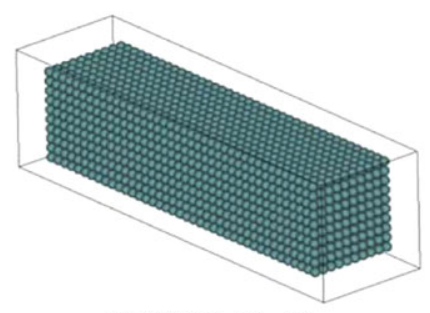

DLSM (Cubic II)

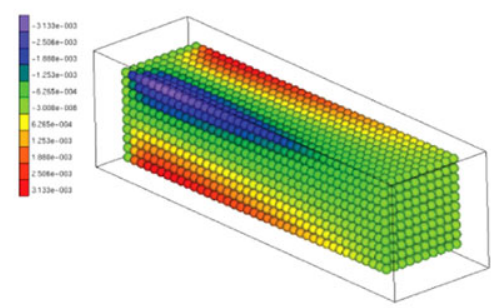

DLSM (Cubic II)
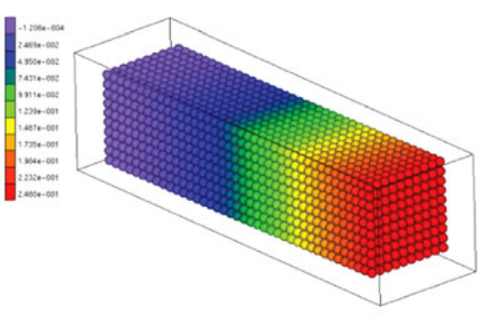

DLSM (Cubic II)
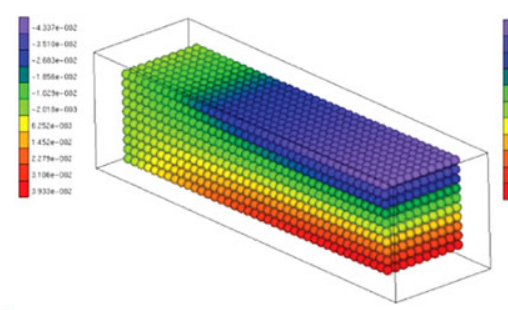

DLSM (Cubic II)

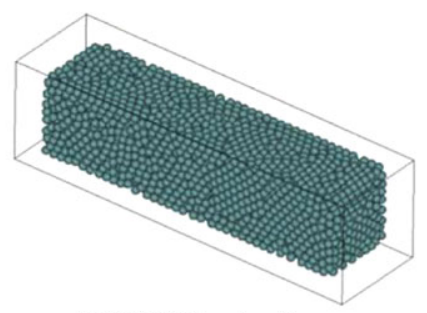

DLSM (Random)
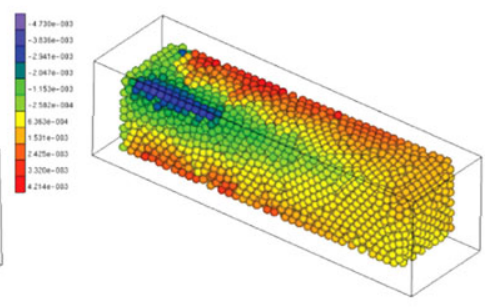

DLSM (Random)
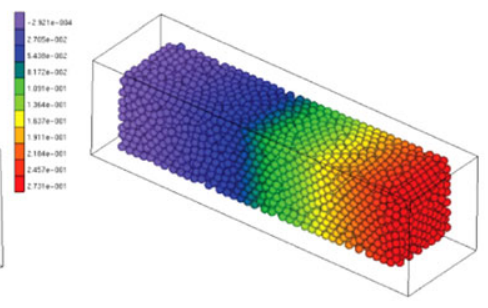

DLSM (Random)
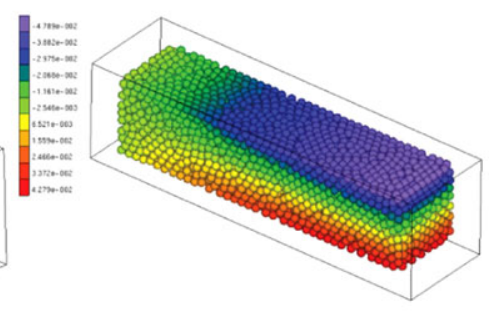

DLSM (Random)

Figure 7. Numerical models (a) and contour plot of the displacement results ( $z$-direction (b); $y$-direction (c) and $x$-direction (d)) predicted by FEM and DLSM for the beam bending problem with Poisson's ratio of 0.2 .

does not correspond to an elastic material. Comparing with the first example, the number of particles plays a very important role in the LSM for accurate modeling of the isotropic elasticity and the relative error here is reduced to $5 \%$. It is important to point out that the simulations have been performed with Poisson's ratio of 0.3, i.e. negative shear springs being used. All of these models are convergent and correct solutions are obtained as demonstrated by the results presented in Figure 8. From the above studies, it can be concluded that the Cubic II structure is the most suitable lattice structure in terms of accuracy and efficiency. This structure is much easier to be generated (compared with the random one) and contains a smaller total number of particles for the same model resolution (defined by the number of divisions in each dimension) compared with the BCC structures. As the contour map cannot give a quantitative comparison 


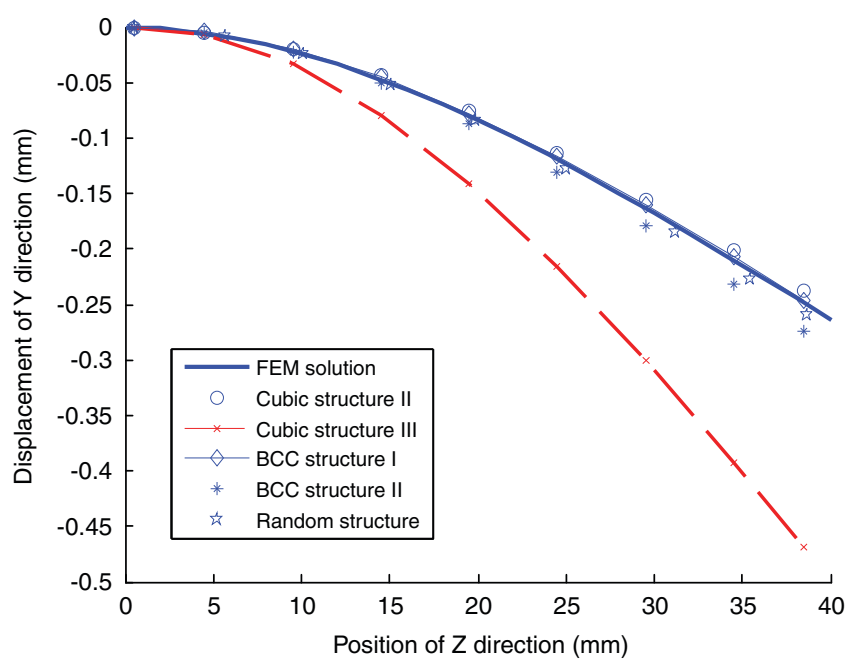

Figure 8 . The $y$-direction displacement along the middle line of the beam predicted by FEM and DLSM with different lattice structures with Poisson's ratio of 0.3 .
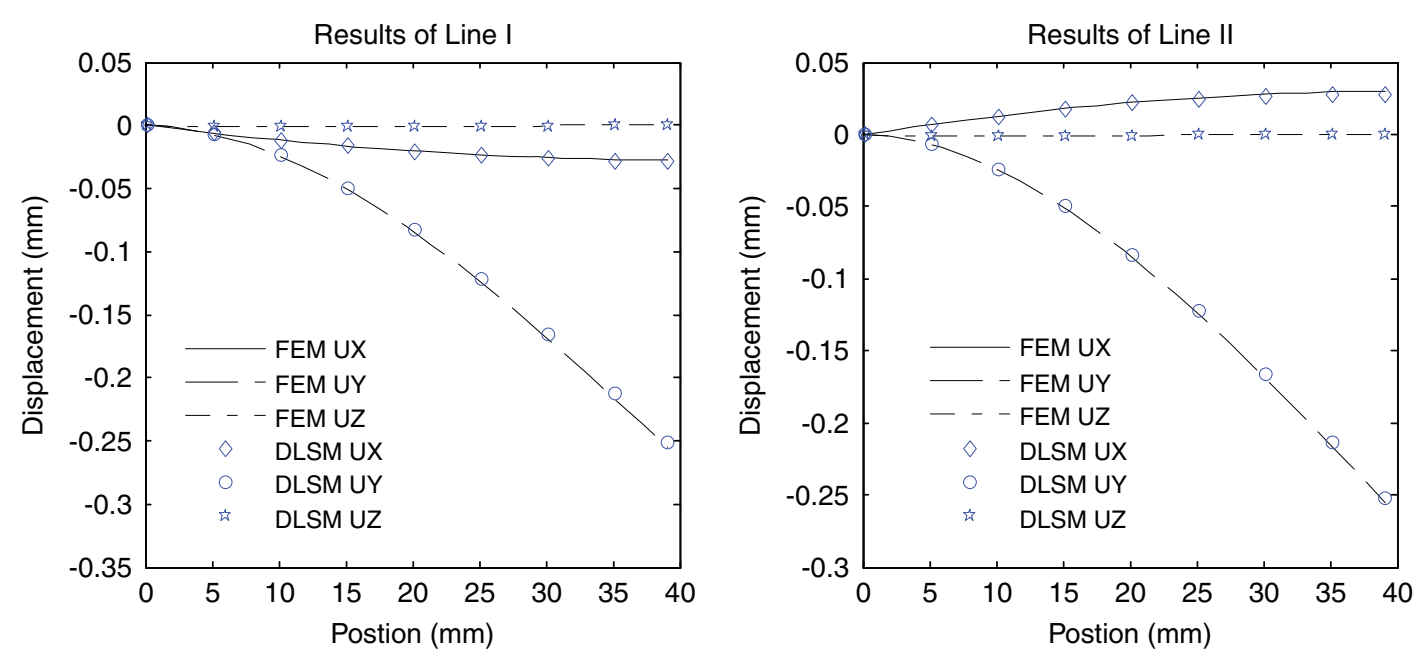

Figure 9. Quantitative comparison of the displacement field predicted by FEM and DLSM.

between the DLSM and FEM results, two section lines, Line I $((0.125,2.125,2.125)-(39.125$, $2.125,2.125))$ and Line II $((0.125,8.125,8.125)-(39.125,8.125,8.125))$, are selected to record displacements predicted by the DLSM model using a higher resolution with particle size of $0.25 \mathrm{~mm}$. A full comparison with the displacement field of FEM is given in Figure 9. It can be seen that the displacement field predicted by DLSM is almost identical to that of FEM model. For the $y$-direction displacement along these two lines, the maximum errors of the DLSM model are 1.53 and $1.13 \%$, respectively. Additionally, a slender beam problem (see Figure 10(a) for detailed problem description) which was solved by using another discrete lattice model in [32] is simulated by DLSM. Particle size used is $1 \mathrm{~mm}$ and the DLSM modeling result is shown in Figure 10(b). The predicted top-end displacement is $3.967 \mathrm{~mm}$ which is $0.82 \%$ stiffer than the analytical solution, whereas the discrete model in [32] with similar resolution provided an error around 10-15\%. For the two beam bending problems, the regular Cubic II structure can predict correct results. However, the DLSM model based on the Cubic II structure is not strictly isotropic because of the regular arrangement of particles and hence the non-uniform distribution of bonds. The influence of this kind 


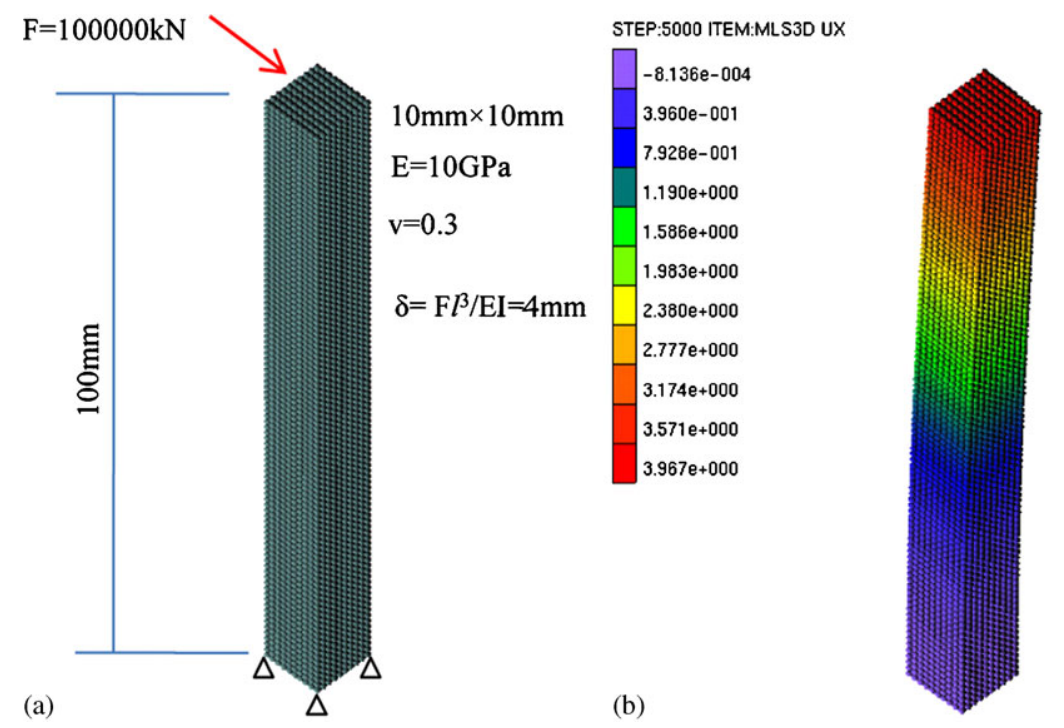

Figure 10. The slender beam problem (a) and the results (b) of the DLSM model.

of anisotropy on the numerical simulation of isotropic elastic materials will be studied in the next example.

\subsection{The braziliar test}

In this section the braziliar disc problem is selected to study the anisotropic effects of the DLSM model. As shown in Figure 11, two DLSM models of two different lattice structures are used for this study. The elastic properties of the modeled isotopic material are $E=10 \mathrm{GPa}$ and $v=0.2$. The average particle diameter of the random lattice model generated by PFC is $7.15 \mathrm{~mm}$ and that of the Cubic II lattice is $5 \mathrm{~mm}$. In order to check the anisotropic behavior of the two DLSM models, the original models are rotated to different angles $\left(10^{\circ}, 30^{\circ}, 45^{\circ}\right)$ as shown in Figure 12 . The strain component $\varepsilon_{x x}$ at the center of the disc is calculated by the rotated models and is compared with the value obtained by the original model. From the results given in Table II, it can be seen that for the random model, the differences caused by the rotation are small (less than $2 \%$ ) and for the Cubic II model the maximum difference happens at the rotation angle of $45^{\circ}$, which reaches $9.2 \%$ for a low space resolution. In this sense, the random model is a more realistic choice for the simulation of isotropic elastic materials. However, as we mention before, the generation of this kind of model is complex and time consuming. Regarding the fact that the Cubic II model with a high resolution $(100 \times 100 \times 10)$ reduces the maximum difference to $5.34 \%$ (see Table II), which is acceptable for practical applications, we recommend it as an alternative choice, especially when efficiency is considered to be prior. The particle size of the high-resolution DLSM model is $1 \mathrm{~mm}$ and the whole model is made up of 100000 particles. This DLSM model is then used to simulate the braziliar disc problem for different Poisson's ratios. The results obtained by different methods, including FEM as a reference solution, are summarized in Table III, where CLSM stands for the classical lattice spring model which directly calculates the deformation of shear springs using the particle displacement. Here, the particle size of DLSM and CLSM are both taken as $1 \mathrm{~mm}$. Cubic II is selected as the lattice type. The elastic module is taken as $10 \mathrm{GPa}$ and the Poisson's ratio is taken as $0.10,0.20,0.25$, and 0.30 . The spring parameters are calculated based on Equation (26) for both DLSM and CLSM. The comparison is used to illustrate the importance of keeping rotation invariance. From the results, it can be seen that the Poisson's ratio has a great influence on the results and the Poisson's ratio dependant mechanical response can be well captured by FEM and DLSM. However, CLSM could only provide reasonable results at the Poisson's ratio of 0.25 when shear spring does not present. This indicates that preserving the rotation invariance is very important for the LSM. 


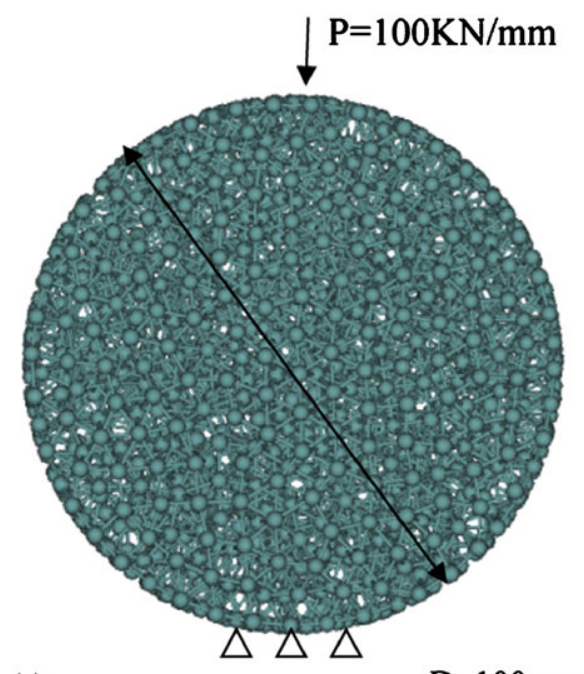

(a)

$$
\mathrm{D}=100 \mathrm{~mm}
$$
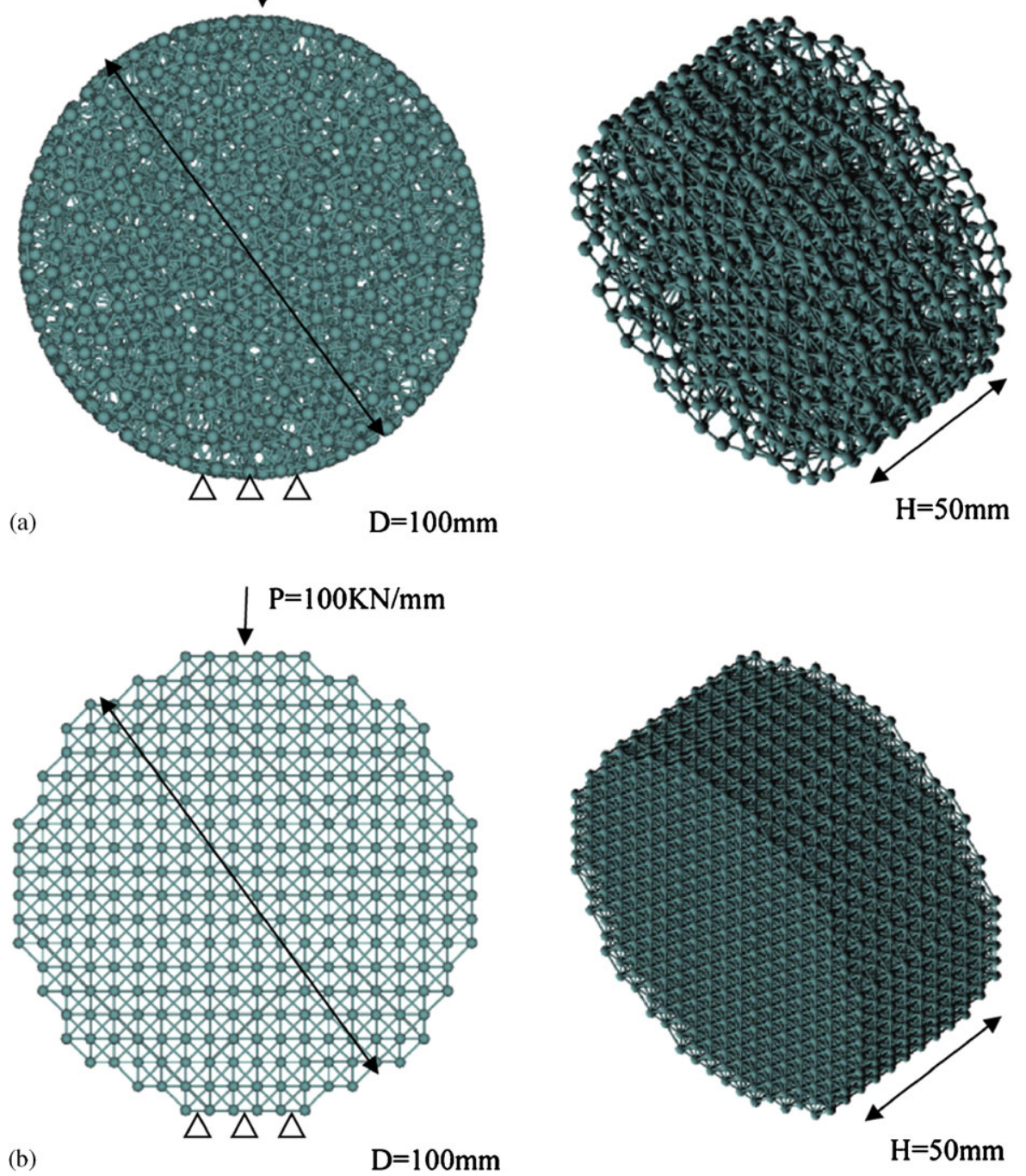

Figure 11. Two lattice structures for the braziliar disc problem: (a) the random lattice structure model (left: plane view) and (b) the Cubic II lattice structure model (left: plane view).

\subsection{Elastic wave propagation}

Wave propagation can be viewed as the transmission of dynamic loads through materials. It is an important research issue in dynamic failure study. For example, the prediction of wave attenuation across fractured rock masses is very important in solving problems in geophysics, seismic investigations, and rock protective engineering. Numerical methods and computing techniques have been proven as a powerful and effective tool to simulate and model rock mechanical problems, for example in the work of Chen and Zhao [33], wave propagation in jointed rock masses was studied through DEM. The following example will show the ability of DLSM in modeling wave propagation in elastic materials. Wave propagation through an assembly of discrete bodies is, in general, dispersive. That is, the apparent wave velocity depends on wavelength, particularly for wavelengths that approach the average particle size. For longer wavelengths, the propagation behaviors, as in a continuous elastic medium, are without an internal length scale. The example illustrates wave propagation through a one-dimensional bar composed of 20000 particles bonded together. The right-hand side of the bar is free and an input pulse is applied at the left-hand boundary. In DLSM, the input parameters are the macroscopic parameters and the microscopic 

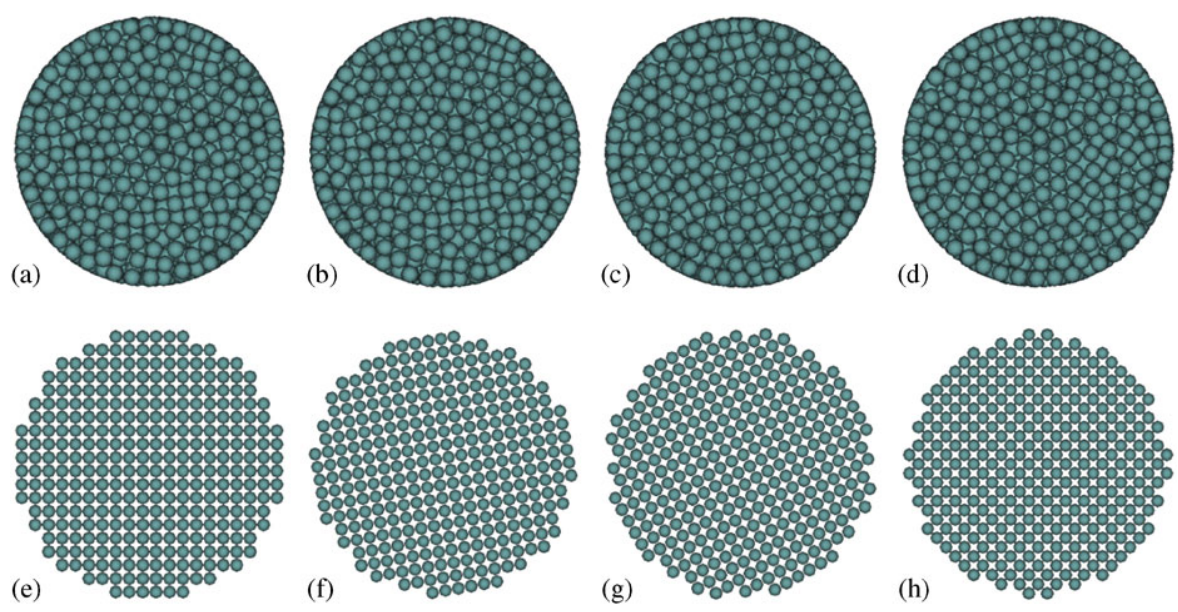

Figure 12. The lattice models with different rotation angles for the braziliar disc problem: (a) Random $0^{\circ}$; (b) Random $10^{\circ}$; (c) Random 30 ; (d) Random 45 ; (e) Cubic II $0^{\circ}$; (f) Cubic II $10^{\circ}$; (g) Cubic II $30^{\circ}$; and (h) Cubic II $45^{\circ}$.

Table II. The predicted $\varepsilon_{x x}$ at the disc center by DLSM models with different rotated angles.

\begin{tabular}{|c|c|c|c|c|c|c|}
\hline \multirow[b]{2}{*}{ Rotation angle $\left({ }^{\circ}\right)$} & \multicolumn{2}{|c|}{ Random model } & \multicolumn{2}{|c|}{ Cubic II model } & \multicolumn{2}{|c|}{ Cubic II model (high resolution) } \\
\hline & $\varepsilon_{x x}^{\text {center }}\left(10^{-6}\right)$ & Error $(\%)$ & $\varepsilon_{x x}^{\text {center }}\left(10^{-6}\right)$ & Error $(\%)$ & $\varepsilon_{x x}^{\text {center }}\left(10^{-6}\right)$ & Error $(\%)$ \\
\hline 0 & 0.0717 & 0.00 & 0.0674 & 0.00 & 0.0749 & 0.00 \\
\hline 10 & 0.0718 & 0.11 & 0.0651 & 3.41 & 0.0738 & 1.47 \\
\hline 30 & 0.0710 & 0.94 & 0.0644 & 4.45 & 0.0738 & 1.47 \\
\hline 45 & 0.0705 & 1.65 & 0.0612 & 9.20 & 0.0709 & 5.34 \\
\hline
\end{tabular}

Table III. The results predicted by FEM, DLSM, and CLSM for the Brazilian disc problem.

\begin{tabular}{lcccccc}
\hline & & \multicolumn{2}{c}{ DLSM } & & \multicolumn{2}{c}{ CLSM } \\
\cline { 3 - 4 } \cline { 5 - 6 } Poisson's ratio & FEM $\left(10^{-6}\right)$ & $\varepsilon_{x x}^{\text {center }}\left(10^{-6}\right)$ & Error $(\%)$ & & $\varepsilon_{x x}^{\text {center }}\left(10^{-6}\right)$ & Error $(\%)$ \\
\hline 0.10 & 0.0794 & 0.0749 & 5.6 & & 0.0207 & 74.0 \\
0.20 & 0.0982 & 0.0941 & 4.1 & & 0.0545 & 44.5 \\
0.25 & 0.1076 & 0.1040 & 3.3 & & 0.1040 & 3.3 \\
0.30 & 0.1170 & 0.1144 & 2.3 & & Unstable & Unstable \\
\hline
\end{tabular}

parameters are automatically computed from Equation (26). In the following calculations, it is assumed that the elastic modulus is $80.461 \mathrm{GPa}$ and the Poisson's ratio is 0.2563 and the rock density is $2600 \mathrm{~kg} / \mathrm{m}^{3}$, which are typical parameters for the Bukit Timah granite. The theoretical wave velocity of $\mathrm{P}$-wave and $\mathrm{S}$-wave is calculated as:

$$
\begin{aligned}
& V_{\mathrm{p}}=\sqrt{\frac{K+4 G / 3}{\rho}}=6128.68 \mathrm{~m} / \mathrm{s} \\
& V_{\mathrm{s}}=\sqrt{G / \rho}=3508.295 \mathrm{~m} / \mathrm{s}
\end{aligned}
$$

where $K$ and $G$ are the bulk and shear stiffness of the material which can be obtained from Equations (16) and (17) and $\rho$ is the density. A half-cycle sinusoidal wave with 1MPa amplitude is applied at the left boundary as the incident wave, where the sinusoidal wave is $50000 \mathrm{~Hz}$. Three detection points $\mathrm{A}(4.5,4.5,49.5), \mathrm{B}(4.5,4.5,99.5), \mathrm{C}(4.5,4.5,149.5)$ are placed in the bar to 

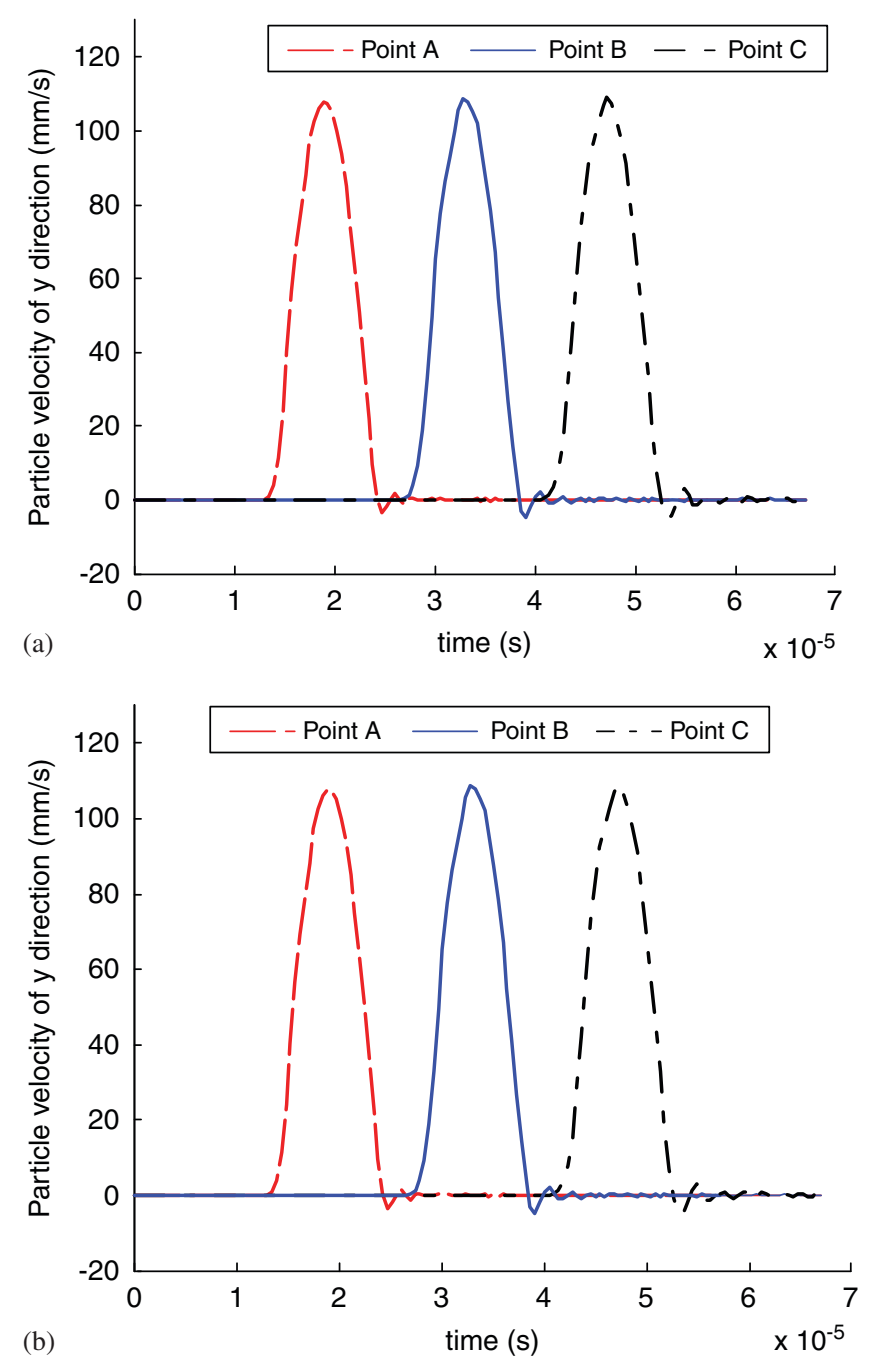

Figure 13. Wave propagation history at the detection points: (a) P-wave and (b) S-wave.

record the wave propagation. Figure 13 shows the propagation of P-wave and S-wave at the three points. From these data we obtain

$$
\begin{aligned}
& \hat{C}_{\mathrm{p}}=\frac{d_{A C}}{t_{A C}^{\mathrm{p}}}=\frac{100 \times 10^{-3}}{2.862 \times 10^{-5}-1.264 \times 10^{-5}}=6257.8 \mathrm{~m} / \mathrm{s} \\
& \hat{C}_{\mathrm{s}}=\frac{d_{A C}}{t_{A C}^{\mathrm{s}}}=\frac{100 \times 10^{-3}}{4.725 \times 10^{-5}-1.890 \times 10^{-5}}=3527.3 \mathrm{~m} / \mathrm{s}
\end{aligned}
$$

where $\hat{C}_{\mathrm{p}}$ and $\hat{C}_{\mathrm{s}}$ are the calculated P-wave speed and S-wave speed. $d_{A C}$ is the distance from A point to $\mathrm{C}$ point. $t_{A C}^{\mathrm{p}}$ and $t_{A C}^{\mathrm{s}}$ are the time used for $\mathrm{P}$-wave and $\mathrm{S}$-wave transmitted from A point to $\mathrm{C}$ point. The error of the calculated speed is $2.11 \%$ for $\mathrm{P}$-wave and $0.54 \%$ for $\mathrm{S}$-wave. It can be concluded that the DLSM could predict correct wave propagation speed. The Poisson's ratio is greater than 0.25 , hence again the model here has negative shear springs involved. This means negative spring is also applicable in DLSM for dynamics problems. Figure 14 shows the contour map of particle velocity. From this figure, the propagation and reflection of the stress wave can be observed clearly. Overall, this example demonstrates that the dynamic behaviors of elastic material 


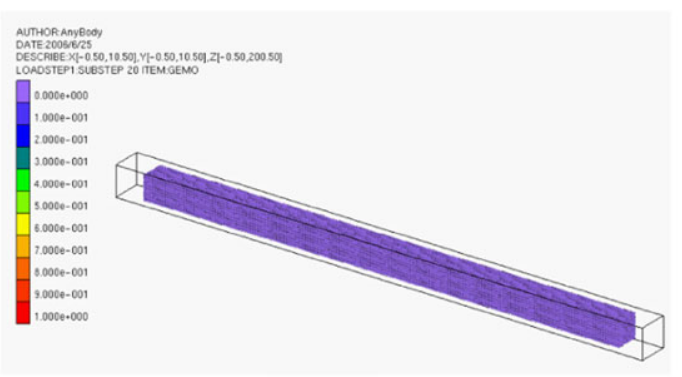

$\mathrm{t}=0 \mu \mathrm{s}$

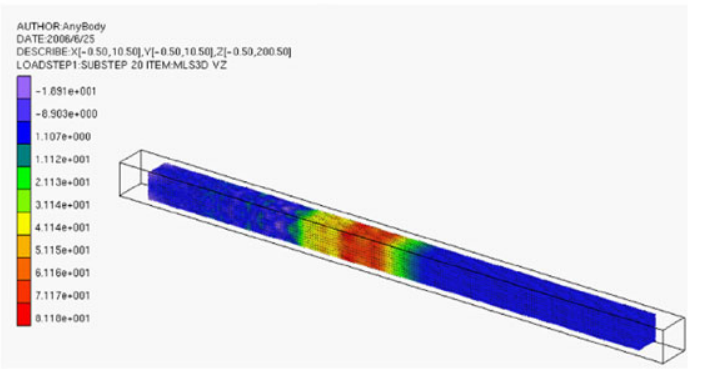

$\mathrm{t}=18 \mu \mathrm{s}$

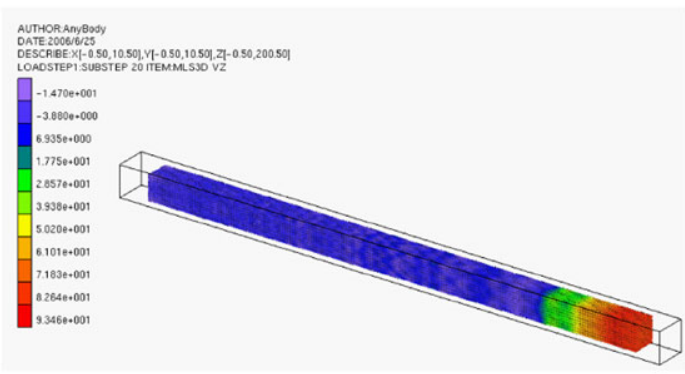

$t=36 \mu \mathrm{s}$

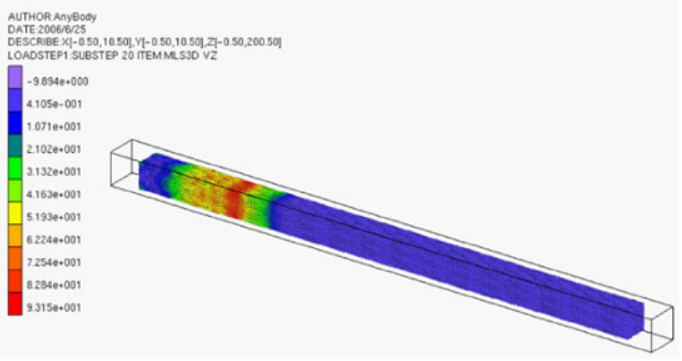

$t=9 \mu \mathrm{s}$

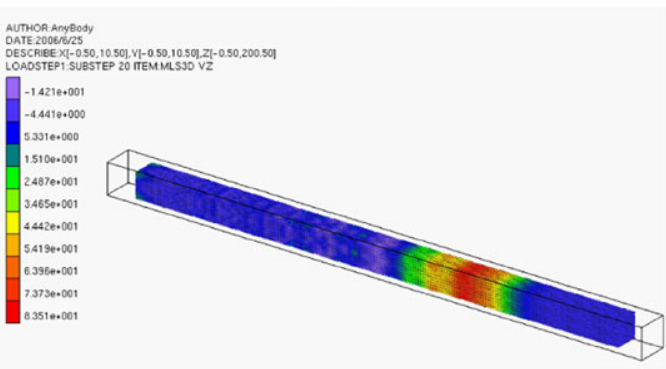

$\mathrm{t}=27 \mu \mathrm{s}$

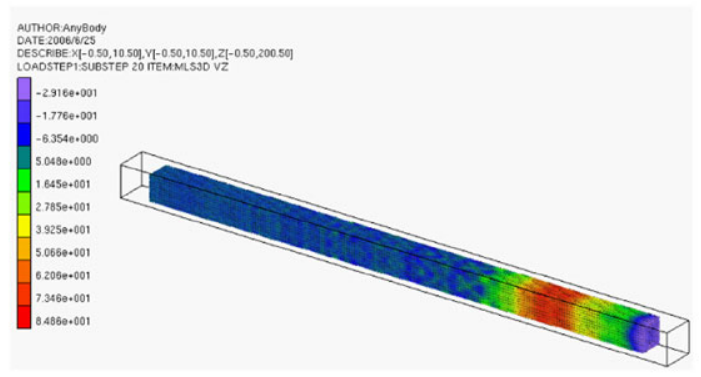

$\mathrm{t}=45 \mu \mathrm{s}$

Figure 14. The process of wave propagation through the elastic bar predicted by DLSM.

could be well predicted by DLSM and gives us confidence to use DLSM to study the dynamic failure behavior of elastic material in the following two examples.

\subsection{Dynamic spalling of $3 D$ bar}

The dynamic spalling of quasi-brittle material occurs when an incident compressive wave is reflected by a free end and transformed into a tensile one. Spalling happens when the inputted incident compressive stress wave is lower than the material compressive strength whereas it is larger than its tensile strength. It has been successfully simulated by the FEM in the work of Zhu and Tang [34]. In this subsection, the ability of DLSM on modeling the dynamic fracturing process will be studied through this example.

The geometries and loading conditions for the bar model are shown in Figure 15. The mechanical properties of the bar are as follows: the Young's modulus is $60.0 \mathrm{GPa}$, the direct tensile strength is $19.0 \mathrm{MPa}$, and the Poisson's ratio is 0.20 . The model is $200 \mathrm{~mm}$ in length, $10 \mathrm{~mm}$ in width and height, and it is discretized with $200 \times 10 \times 10$ particles. The incident compressive stress wave is applied at the left end of the bar. The right end is kept free during calculation. Other faces are fixed in their normal directions. Two cases were simulated. Different compressive incident waves were applied on the left surface of the model (as shown in Figure 15) to study the stress amplitude influence on the spalling failure. For the incident compressive stress wave I ( $p_{\max }=20 \mathrm{MPa}$ ), a fracture face located at $24 \mathrm{~mm}$ from the right end of the model is detected (see Figure 16). 


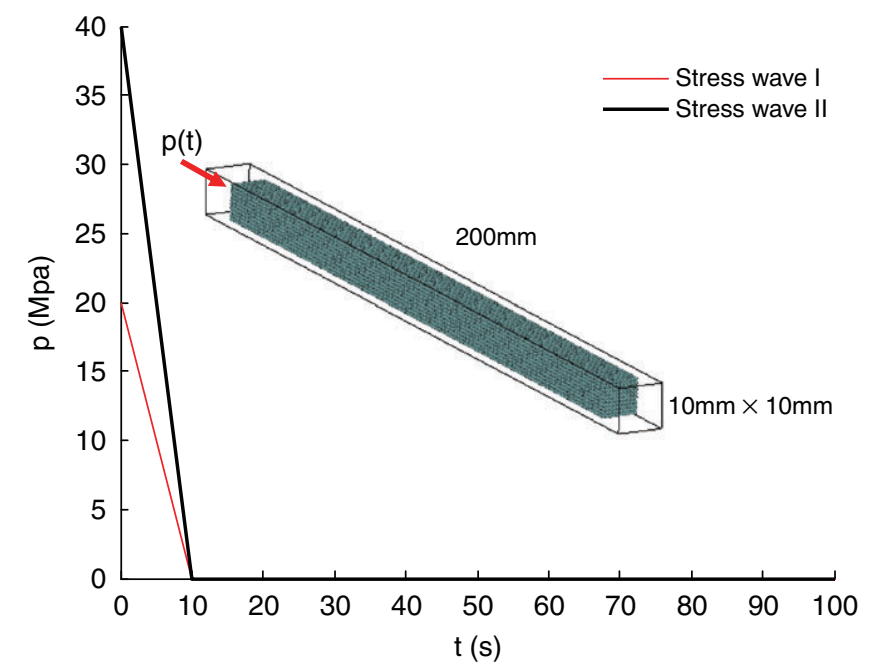

Figure 15. The incident compressive stress waves with different peak amplitudes applied on the left surface of the bar.

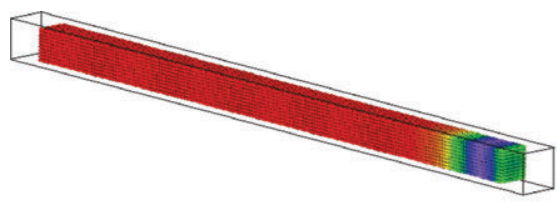

$\mathrm{t}=40.2 \mu \mathrm{s}$

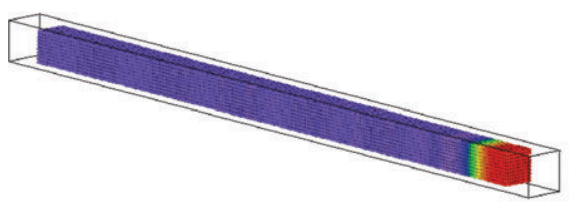

$\mathrm{t}=42 \mu \mathrm{s}$

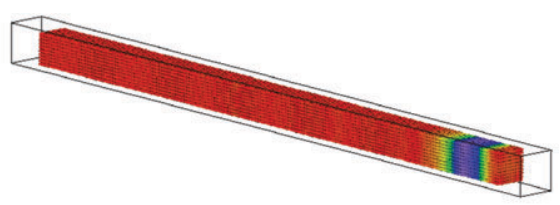

$\mathrm{t}=40.8 \mu \mathrm{s}$

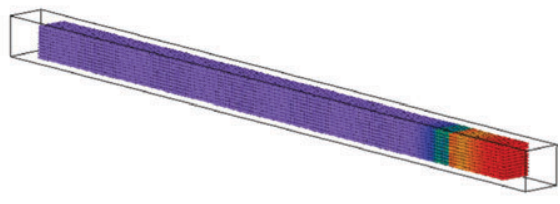

$\mathrm{t}=45 \mu \mathrm{s}$

Failure surface at $24 \mathrm{~mm}$ from the end.

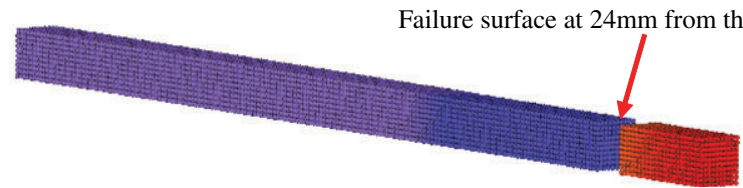

Figure 16. Dynamic spalling predicted by DLSM (incident compressive stress wave of $20 \mathrm{MPa}$ ).

The principle behind this phenomenon can be explained as follows. First, the compressive stress wave travels through the bar and reaches the right end. Because of the wave reflection, the original compressive stress wave will be transformed into a tensile stress wave which eventually induces the failure. After this, the strain energy is released and the stress amplitude of the residual wave is not large enough anymore to cause further failure.

For the incident compressive stress wave II $\left(p_{\max }=40 \mathrm{MPa}\right)$ where the peak value of the stress is twice bigger than the tensile strength of the material, two apparent successive spalling failures occur (see Figure 17). The first fracture surface happens at $12 \mathrm{~mm}$ from the right end of the model. After failure, the yielded surface reduces a portion of the original wave (see Figure 17). Meanwhile, the fraction of the stress wave that has passed the first failure surface continues traveling along the specimen. Because its magnitude is still larger than the tensile strength of the material, another spalling failure happens. The second failure surface occurs at $25 \mathrm{~mm}$ from 

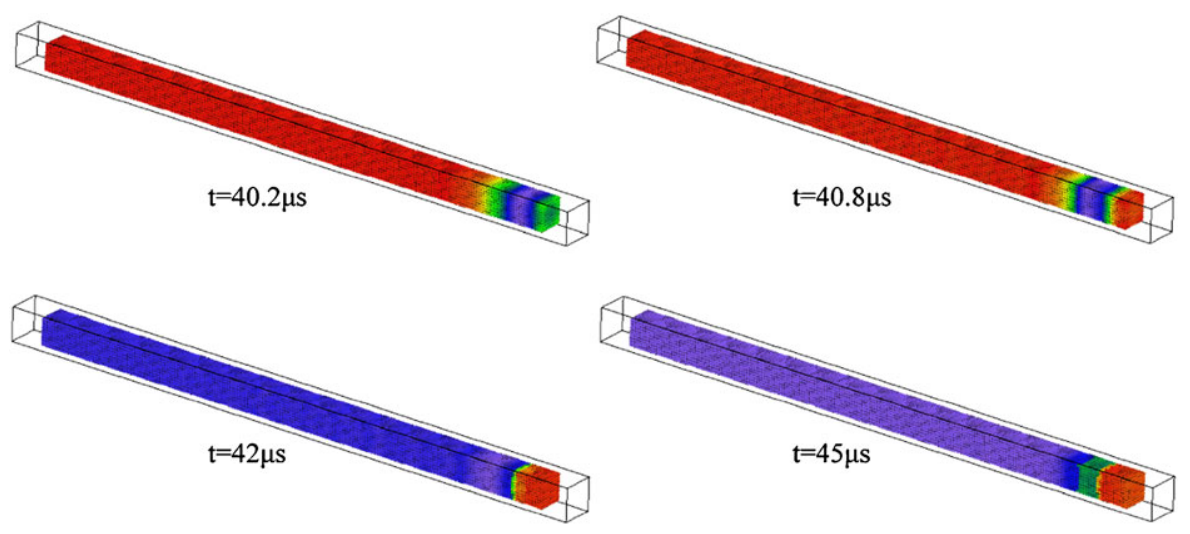

First failure surface at $12.0 \mathrm{~mm}$ from the end

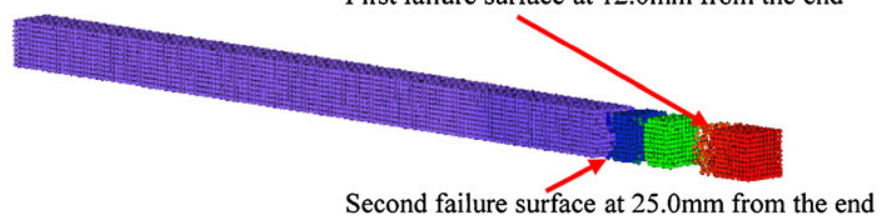

Figure 17. Dynamic spalling predicted by DLSM (incident compressive stress wave of $40 \mathrm{MPa}$ ).

Table IV. The spalling results predicted by DLSM.

\begin{tabular}{lcccccc}
\hline & \multicolumn{2}{c}{ Number of spalling } & & \multicolumn{2}{c}{ Distance from the specimen end (mm) } \\
\cline { 2 - 3 } & Theoretical & DLSM & & Theoretical & DLSM & Relative error \\
\hline Stress wave I & 1 & 1 & & 24.50 & 24 & $2.0 \%$ \\
Stress wave II & 2 & 2 & & 12.25 & 12 & $2.0 \%$ \\
& & & 24.50 & 25 & $2.0 \%$ \\
\hline
\end{tabular}

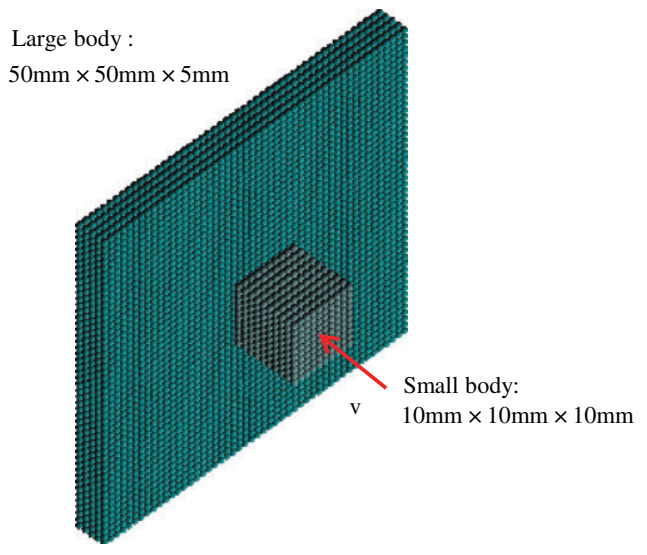

Figure 18. Diagram for the collision of two bodies.

the right end of the model. After this, the residual stress wave is not strong enough to fracture the material. The simulation results of DLSM are compared with the theoretical solutions based on 1D wave propagation theory [35] and the experimental observations [36] (see Table IV). Based on these results, it can be concluded that the DLSM is able to model the dynamic failure process of brittle materials, e.g. rock and concrete, under dynamic loading. 
Table V. Parameter values for the simulation of the collision problem.

\begin{tabular}{lcccc}
\hline & Elastic modules $(\mathrm{GPa})$ & Poisson's ratio & Density $\left(\mathrm{kg} / \mathrm{m}^{3}\right)$ & Strength $(\mathrm{MPa})$ \\
\hline Small body & 120 & 0.2 & 7900 & 30000 \\
Large body & 60 & 0.2 & 2500 & $16 / 0.59 / 0.16$ \\
\hline
\end{tabular}

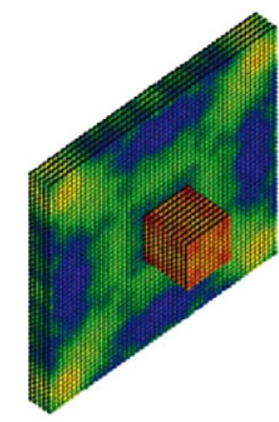

(a) $\quad 10.2 \mathrm{~ms}$

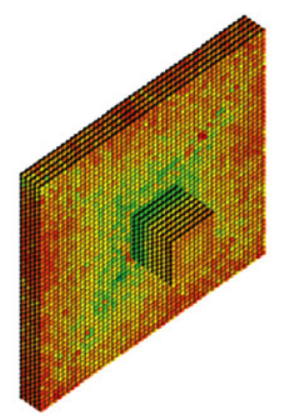

(b) $10.2 \mathrm{~ms}$

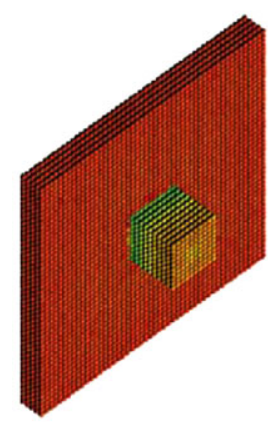

(c) $10.2 \mathrm{~ms}$

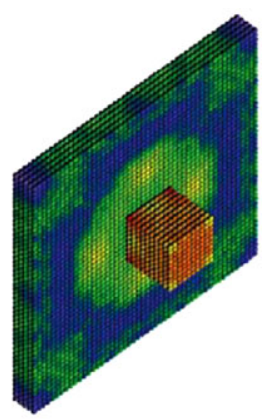

$11.0 \mathrm{~ms}$

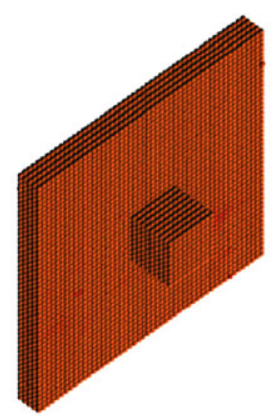

$11.0 \mathrm{~ms}$

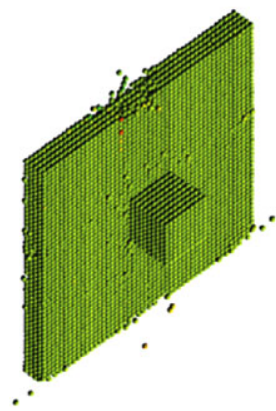

$11.0 \mathrm{~ms}$

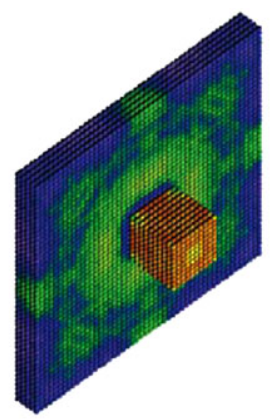

$11.2 \mathrm{~ms}$

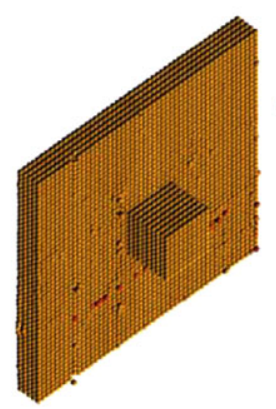

$11.2 \mathrm{~ms}$

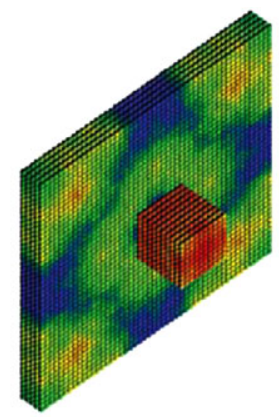

$12.0 \mathrm{~ms}$

Figure 19. DLSM simulation of the dynamic failure process of the large body impacted by the intruder: (a) large body with strength of $16 \mathrm{MPa}$; (b) large body with strength of $0.59 \mathrm{MPa}$; and (c) large body with strength of $0.16 \mathrm{MPa}$.

\subsection{Collision of two bodies}

The collision of two bodies made from different materials is selected as another example to illustrate the simulation of dynamic failure using DLSM. A sketch of the initial configuration for the simulation is shown in Figure 18. The smaller body (called intruder here) strikes the large body with a high velocity. At the beginning of the simulation, the two bodies are formed using $10 \times 10 \times 10$ and $50 \times 50 \times 5$ particles, respectively. The velocity of the intruder is initially set to $\mathbf{v}=100 \mathrm{~mm} / \mathrm{s}$. Table V shows the parameter values for the simulation. The strength of the large 
body is set to different values to study the effect of the strength on the collision results. The elastic parameters are chosen in such a way that the two bodies are rather stiff. Figure 19 shows the results of the simulation. The color of each particle represents its velocity in $z$-direction. It is found that immediately after impact shock waves start to spread through the larger body. They first appear on the surface and then propagate into the internal part of the body. In the first case (see Figure 19(a)), the larger body keeps intact after collision. In the latter two cases (Figures 19(b, c)), the larger body is completely destroyed under the impact of the small body. Because of the simple fracture criterion used in the simulation, the results only qualitatively demonstrate the collision of solid bodies. More realistic and quantitative simulation can be accomplished if more advanced micro failure laws are implemented.

\section{CONCLUSIONS}

This paper presents a novel 3D LSM, in which the deformation of shear springs is calculated by using the local strain instead of the particle displacement. It has been proven that this novelty makes the model rotationally invariant and be capable of representing the diversity of Poisson's ratio. Based on the Cauchy-Born rules, the relationship which bridges the spring parameters and the elastic constants is derived. Several numerical examples are presented to show that the proposed model is capable of modeling the elasticity, wave propagation, and dynamic failure. For Poisson's ratio greater than $1 / 4$, the model with negative shear springs still produces reasonable results for both static and dynamic cases as demonstrated numerically. The DLSM model has advantages of directly using macroscopic parameters and allowing general lattice structures to be adopted. The disadvantage of the proposed model is that a local strain has to be calculated which costs more computing resources than the classical lattice model does. Generally speaking, like other discrete models based on the minimum potential energy principle, this model gives a stiff approximation of the corresponding elastic solution. The proposed model supplies an alternative numerical tool for studying the microstructure influences on dynamic fracturing of geo-materials, such as rock and concrete.

\section{APPENDIX A}

In this appendix, Equation (7) used for evaluating the deformation of shear springs in DLSM is derived. First, consider a cubic unit volume containing a bond connecting two particles as shown in Figure A1. The complete first-order displacement function of the cubic is

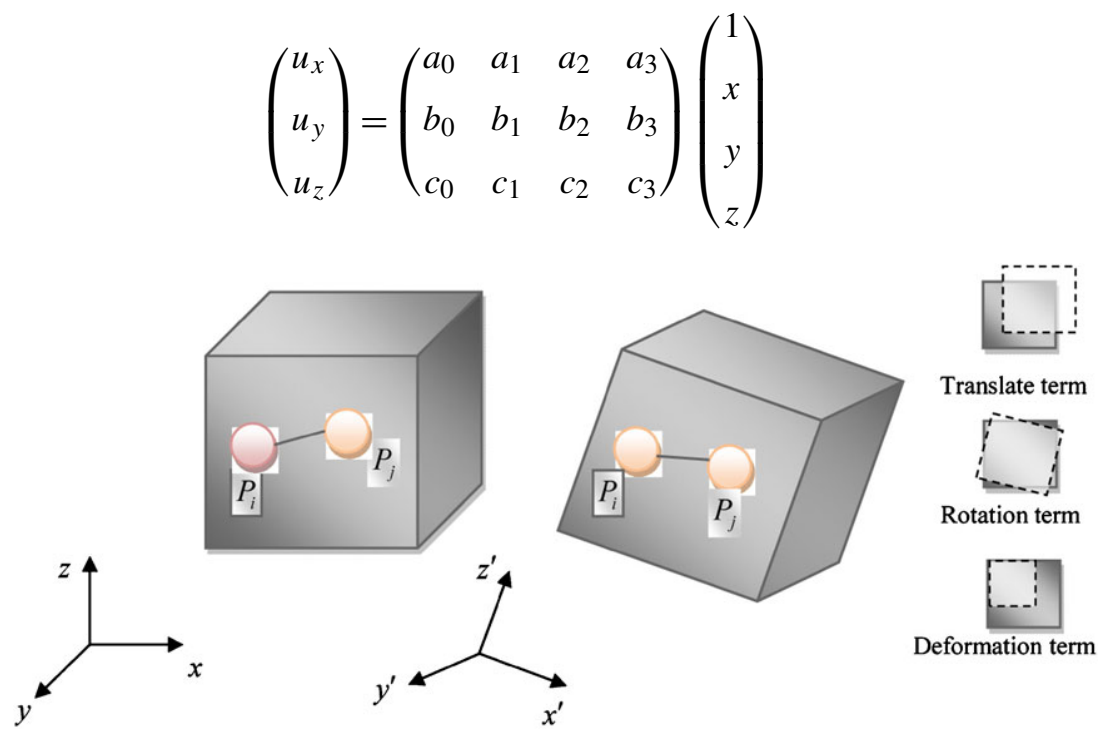

Figure A1. Illustration of the deformation of a cubic unit with a bond connecting two particles. 
Assuming the center of the block at $\left(x_{c}, y_{c}, z_{c}\right)$, then its displacement is represented by

$$
\left(\begin{array}{l}
u_{c x} \\
u_{c y} \\
u_{c z}
\end{array}\right)=\left(\begin{array}{llll}
a_{0} & a_{1} & a_{2} & a_{3} \\
b_{0} & b_{1} & b_{2} & b_{3} \\
c_{0} & c_{1} & c_{2} & c_{3}
\end{array}\right)\left(\begin{array}{c}
1 \\
x_{c} \\
y_{c} \\
z_{c}
\end{array}\right)
$$

Subtracting (A2) from (A1) gives

$$
\left(\begin{array}{l}
u_{x} \\
u_{y} \\
u_{z}
\end{array}\right)-\left(\begin{array}{l}
u_{c x} \\
u_{c y} \\
u_{c z}
\end{array}\right)=\left(\begin{array}{llll}
a_{0} & a_{1} & a_{2} & a_{3} \\
b_{0} & b_{1} & b_{2} & b_{3} \\
c_{0} & c_{1} & c_{2} & c_{3}
\end{array}\right)\left(\begin{array}{l}
1 \\
x \\
y \\
z
\end{array}\right)-\left(\begin{array}{llll}
a_{0} & a_{1} & a_{2} & a_{3} \\
b_{0} & b_{1} & b_{2} & b_{3} \\
c_{0} & c_{1} & c_{2} & c_{3}
\end{array}\right)\left(\begin{array}{c}
1 \\
x_{c} \\
y_{c} \\
z_{c}
\end{array}\right)
$$

Equation (A3) can be further written as

$$
\left(\begin{array}{l}
u_{x} \\
u_{y} \\
u_{z}
\end{array}\right)=\left(\begin{array}{l}
u_{c x} \\
u_{c y} \\
u_{c z}
\end{array}\right)+\left(\begin{array}{ccc}
a_{1} & 0 & 0 \\
0 & b_{2} & 0 \\
0 & 0 & c_{3}
\end{array}\right)\left(\begin{array}{l}
x-x_{c} \\
y-y_{c} \\
z-z_{c}
\end{array}\right)+\left(\begin{array}{ccc}
0 & a_{2} & a_{3} \\
b_{1} & 0 & b_{3} \\
c_{1} & c_{2} & 0
\end{array}\right)\left(\begin{array}{l}
x-x_{c} \\
y-y_{c} \\
z-z_{c}
\end{array}\right)
$$

From (A4), we have

$$
\begin{gathered}
\varepsilon_{x x}=\frac{\partial u_{x}}{\partial x}=a_{1} \\
\varepsilon_{y y}=\frac{\partial u_{y}}{\partial y}=b_{2} \\
\varepsilon_{z z}=\frac{\partial u_{z}}{\partial z}=c_{3} \\
\omega_{x}=\frac{1}{2}\left(\frac{\partial u_{z}}{\partial y}-\frac{\partial u_{y}}{\partial z}\right)=\frac{1}{2}\left(c_{2}-b_{3}\right) \\
\omega_{y}=\frac{1}{2}\left(\frac{\partial u_{x}}{\partial z}-\frac{\partial u_{z}}{\partial x}\right)=\frac{1}{2}\left(a_{3}-c_{1}\right) \\
\omega_{z}=\frac{1}{2}\left(\frac{\partial u_{y}}{\partial x}-\frac{\partial u_{x}}{\partial y}\right)=\frac{1}{2}\left(b_{1}-a_{2}\right) \\
\varepsilon_{y z}=\frac{1}{2}\left(\frac{\partial u_{z}}{\partial y}+\frac{\partial u_{y}}{\partial z}\right)=\frac{1}{2}\left(c_{2}+b_{3}\right) \\
\varepsilon_{z x}=\frac{1}{2}\left(\frac{\partial u_{x}}{\partial z}+\frac{\partial u_{z}}{\partial x}\right)=\frac{1}{2}\left(a_{3}+c_{1}\right) \\
\varepsilon_{x y}=\frac{1}{2}\left(\frac{\partial u_{y}}{\partial x}+\frac{\partial u_{x}}{\partial y}\right)=\frac{1}{2}\left(b_{1}+a_{2}\right)
\end{gathered}
$$

Using the above relations, Equation (A4) can be transformed into

$$
\left(\begin{array}{l}
u_{x} \\
u_{y} \\
u_{z}
\end{array}\right)=\left[D_{0}\right]\left(\begin{array}{l}
u_{c x} \\
u_{c y} \\
u_{c z}
\end{array}\right)+\left[D_{1}\right]\left(\begin{array}{c}
\omega_{x} \\
\omega_{y} \\
\omega_{z}
\end{array}\right)+\left[D_{2}\right]\left(\begin{array}{c}
\varepsilon_{x x} \\
\varepsilon_{y y} \\
\varepsilon_{z z}
\end{array}\right)+\left[D_{3}\right]\left(\begin{array}{c}
\varepsilon_{y z} \\
\varepsilon_{z x} \\
\varepsilon_{x y}
\end{array}\right)
$$


where

$$
\begin{aligned}
{\left[D_{0}\right] } & =\left(\begin{array}{lll}
1 & 0 & 0 \\
0 & 1 & 0 \\
0 & 0 & 1
\end{array}\right) \\
{\left[D_{1}\right] } & =\left(\begin{array}{ccc}
0 & z-z_{c} & -\left(y-y_{c}\right) \\
-\left(z-z_{c}\right) & 0 & x-x_{c} \\
y-y_{c} & -\left(x-x_{c}\right) & 0
\end{array}\right) \\
{\left[D_{2}\right] } & =\left(\begin{array}{ccc}
x-x_{c} & 0 & 0 \\
0 & y-y_{c} & 0 \\
0 & 0 & z-z_{c}
\end{array}\right) \\
{\left[D_{3}\right] } & =\left(\begin{array}{ccc}
0 & z-z_{c} & y-y_{c} \\
z-z_{c} & 0 & x-x_{c} \\
y-y_{c} & x-x_{c} & 0
\end{array}\right)
\end{aligned}
$$

Denoting the coordinates of the two particles in the cubic as $\left(x_{1}, y_{1}, z_{1}\right)$ and $\left(x_{2}, y_{2}, z_{2}\right)$ and the displacement of them as $\left(u_{1}, v_{1}, w_{1}\right)$ and $\left(u_{2}, v_{2}, w_{2}\right)$, the relative displacement vector between the two particles is

$$
\left(\begin{array}{l}
u_{12 x} \\
u_{12 y} \\
u_{12 z}
\end{array}\right)=\left(\begin{array}{l}
u_{2 x}-u_{1 x} \\
u_{2 y}-u_{1 y} \\
u_{2 z}-u_{1 z}
\end{array}\right)
$$

and the normal unit vector is

$$
\left(\begin{array}{l}
n_{x} \\
n_{y} \\
n_{z}
\end{array}\right)=\left(\begin{array}{l}
\frac{x_{2}-x_{1}}{l} \\
\frac{y_{2}-y_{1}}{l} \\
\frac{z_{2}-z_{1}}{l}
\end{array}\right)
$$

where $l$ is the length of the bond. The relative normal displacement vector is defined as

$$
\left(\begin{array}{l}
u_{12 x}^{\mathrm{n}} \\
u_{12 y}^{\mathrm{n}} \\
u_{12 z}^{\mathrm{n}}
\end{array}\right)=\left(\left(\begin{array}{l}
u_{12 x} \\
u_{12 y} \\
u_{12 z}
\end{array}\right)^{\mathrm{T}}\left(\begin{array}{l}
n_{x} \\
n_{y} \\
n_{z}
\end{array}\right)\right)\left(\begin{array}{l}
n_{x} \\
n_{y} \\
n_{z}
\end{array}\right)
$$

By vector operation, the relative shear displacement vector is obtained as

$$
\left(\begin{array}{l}
u_{12 x}^{\mathrm{s}} \\
u_{12 y}^{\mathrm{s}} \\
u_{12 z}^{\mathrm{s}}
\end{array}\right)=\left(\begin{array}{l}
u_{12 x} \\
u_{12 y} \\
u_{12 z}
\end{array}\right)-\left(\begin{array}{l}
u_{12 x}^{\mathrm{n}} \\
u_{12 y}^{\mathrm{n}} \\
u_{12 z}^{\mathrm{n}}
\end{array}\right)=\left(\begin{array}{l}
u_{12 x} \\
u_{12 y} \\
u_{12 z}
\end{array}\right)-\left(\left(\begin{array}{l}
u_{12 x} \\
u_{12 y} \\
u_{12 z}
\end{array}\right)^{\mathrm{T}}\left(\begin{array}{l}
n_{x} \\
n_{y} \\
n_{z}
\end{array}\right)\right)\left(\begin{array}{l}
n_{x} \\
n_{y} \\
n_{z}
\end{array}\right)
$$


Now, applying Equation (A5), the relative displacement vector can be represented as

$$
\begin{aligned}
\left(\begin{array}{c}
u_{2 x}-u_{1 x} \\
u_{2 y}-u_{1 y} \\
u_{2 z}-u_{1 z}
\end{array}\right)= & \underbrace{\left(\begin{array}{ccc}
x_{2}-x_{1} & 0 & 0 \\
0 & y_{2}-y_{1} & 0 \\
0 & 0 & z_{2}-z_{1}
\end{array}\right)\left(\begin{array}{c}
\varepsilon_{x x} \\
\varepsilon_{y y} \\
\varepsilon_{z z}
\end{array}\right)+\left(\begin{array}{ccc}
0 & z_{2}-z_{1} & y_{2}-y_{1} \\
z_{2}-z_{1} & 0 & x_{2}-x_{1} \\
y_{2}-y_{1} & x_{2}-x_{1} & 0
\end{array}\right)\left(\begin{array}{c}
\varepsilon_{y z} \\
\varepsilon_{z x} \\
\varepsilon_{x y}
\end{array}\right)}_{\text {strain-related term }} \\
& +\underbrace{\left(\begin{array}{ccc}
0 & z_{2}-z_{1} & -\left(y_{2}-y_{1}\right) \\
-\left(z_{2}-z_{1}\right) & 0 & x_{2}-x_{1} \\
y_{2}-y_{1} & -\left(x_{2}-x_{1}\right) & 0
\end{array}\right)\left(\begin{array}{l}
\omega_{x} \\
\omega_{y} \\
\omega_{z}
\end{array}\right)}_{\text {rotation-related term }}
\end{aligned}
$$

With the above equation, it is straightforward to show that the relative normal displacement vector is only dependant on the strain-related term because of the following equivalence:

$$
\left(\begin{array}{ccc}
0 & z_{2}-z_{1} & -\left(y_{2}-y_{1}\right) \\
-\left(z_{2}-z_{1}\right) & 0 & x_{2}-x_{1} \\
y_{2}-y_{1} & -\left(x_{2}-x_{1}\right) & 0
\end{array}\right)\left(\begin{array}{l}
x_{2}-x_{1} \\
y_{2}-y_{1} \\
z_{2}-z_{1}
\end{array}\right)=0
$$

However, for the relative shear displacement vector, if we directly substitute (A10) into (A9), the rotation-related term will not vanish. It is known that rigid rotation of the cubic should not produce strain energy. Therefore, in DLSM, the rotation-related term is removed from the calculation of the relative shear displacement vector, namely, the relative displacement vector in (A9) is not calculated anymore by using (A10) or (A6), but by the following:

$$
\begin{aligned}
\left(\begin{array}{l}
\hat{u}_{12 x} \\
\hat{u}_{12 y} \\
\hat{u}_{12 z}
\end{array}\right) & =\left(\begin{array}{ccc}
x_{2}-x_{1} & 0 & 0 \\
0 & y_{2}-y_{1} & 0 \\
0 & 0 & z_{2}-z_{1}
\end{array}\right)\left(\begin{array}{l}
\varepsilon_{x x} \\
\varepsilon_{y y} \\
\varepsilon_{z z}
\end{array}\right)+\left(\begin{array}{ccc}
0 & z_{2}-z_{1} & y_{2}-y_{1} \\
z_{2}-z_{1} & 0 & x_{2}-x_{1} \\
y_{2}-y_{1} & x_{2}-x_{1} & 0
\end{array}\right)\left(\begin{array}{l}
\varepsilon_{y z} \\
\varepsilon_{z x} \\
\varepsilon_{x y}
\end{array}\right) \\
& =\left(\begin{array}{ccc}
\varepsilon_{x x} & \varepsilon_{x y} & \varepsilon_{z x} \\
\varepsilon_{x y} & \varepsilon_{y y} & \varepsilon_{y z} \\
\varepsilon_{z x} & \varepsilon_{y z} & \varepsilon_{z z}
\end{array}\right)\left(\begin{array}{l}
x_{2}-x_{1} \\
y_{2}-y_{1} \\
z_{2}-z_{1}
\end{array}\right)
\end{aligned}
$$

Writing (A11) in the vector form, we get

$$
\hat{\mathbf{u}}_{i j}=[\varepsilon] \cdot \mathbf{n} l
$$

Finally, the relative shear displacement vector (the vector form of (A9)) can be written as

$$
\hat{\mathbf{u}}_{i j}^{\mathrm{s}}=[\varepsilon] \cdot \mathbf{n} l-(([\varepsilon] \cdot \mathbf{n} l) \cdot \mathbf{n}) \mathbf{n}
$$

which is Equation (7) in the context.

Moreover, consider one rigid body rotation defined by

$$
\mathbf{u}(\mathbf{x})=\omega \times \mathbf{x}
$$

where $\omega$ is the angular displacement vector with components $\left[\omega_{x}, \omega_{y}, \omega_{z}\right]^{\mathrm{T}}$. By simple derivation, the true gradient of this displacement field is found to be

$$
\nabla \mathbf{u}=\left(\begin{array}{ccc}
0 & -\omega_{z} & \omega_{y} \\
\omega_{z} & 0 & -\omega_{x} \\
-\omega_{y} & \omega_{x} & 0
\end{array}\right)
$$


It is obvious that the strain tensor $\varepsilon=\left(\nabla \mathbf{u}+\nabla \mathbf{u}^{\mathrm{T}}\right) / 2$ vanishes given the skew nature of $\nabla \mathbf{u}$. The least square approximation adopted in DLSM to calculate the gradient of the displacement field is first-order consistent, i.e. it is able to reproduce any linear function and its gradient, so the correct skew nature of $\nabla \mathbf{u}$ is kept numerically. Therefore, the calculated strain $\varepsilon$ is also invariant with respect to the rigid body rotation.

Overall, it is ensured that the DLSM is rotationally invariant in the sense that the strain energy is independent of rigid rotation.

\section{ACKNOWLEDGEMENTS}

Financial support from the China Scholarship Council to the first author is gratefully acknowledged. The research is also partially supported by the Swiss National Science Foundation (200021-116536).

\section{REFERENCES}

1. Bažant ZP, Tabbara MR, Kazemi MT, Pijaudier-Cabot G. Random particle model for fracture of aggregate or fiber composites. Journal of Engineering Mechanics 1990; 116(8):1686-1705.

2. Ostoja-Starzewski M. Lattice models in micromechanics. Applied Mechanics Reviews 2002; 55(1):35-59.

3. Buxton GA, Care CM, Cleaver DJ. A lattice spring model of heterogeneous materials with plasticity. Modelling and Simulation in Materials Science and Engineering 2001; 9(6):485-497.

4. Ostoja-Starzewski M, Sheng PY, Jasiuk I. Damage patterns and constitutive response of random matrix-inclusion composites. Engineering Fracture Mechanics 1997; 58(5-6):581-606.

5. Beale PD, Srolovitz DJ. Elastic fracture in random materials. Physical Review B 1988; 37(10):5500-5507.

6. Srolovitz DJ, Beale PD. Computer simulation of failure in an elastic model with randomly distributed defects. Journal of the American Ceramic Society 1988; 71(5):362-369.

7. Nayfeh AH, Hefzy MS. Continuum modeling of three-dimensional truss-like space structures. AIAA Journal 1978; 16(8):779-787.

8. Donze F, Magnier SA. Formulation of a 3-D numerical model of brittle behaviour. Geophysical Journal International 1995; 122(3):790-802.

9. Kawai T. New discrete models and their application to seismic response analysis of structures. Nuclear Engineering and Design 1978; 48(1):207-229.

10. Zubelewicz A, Bažant ZP. Interface element modeling of fracture in aggregate composites. Journal of Engineering Mechanics 1987; 113(11):1619-1630.

11. Griffiths DV, Mustoe GGW. Modelling of elastic continua using a grillage of structural elements based on discrete element concepts. International Journal for Numerical Methods in Engineering 2001; 50(7):1759-1775.

12. Cusatis G, Bažant ZP, Cedolin L. Confinement-shear lattice model for concrete damage in tension and compression: I. theory. Journal of Engineering Mechanics 2003; 129(12):1439-1448.

13. Lilliu G, van Mier JGM. 3D lattice type fracture model for concrete. Engineering Fracture Mechanics 2003; 70:927-941.

14. Schlangen E, Garboczi EJ. Fracture simulations of concrete using lattice models: computational aspects. Engineering Fracture Mechanics 1997; 57:319-332.

15. Karihaloo BL, Shao PF, Xiao QZ. Lattice modelling of the failure of particle composites. Engineering Fracture Mechanics 2003; 70(17):2385-2406.

16. Liu JX, Deng SC, Zhang J, Liang NG. Lattice type of fracture model for concrete. Theoretical and Applied Fracture Mechanics 2007; 48(3):269-284.

17. Hassold GN, Srolovitz DJ. Brittle fracture in materials with random defects. Physical Review B 1989; 39(13):9273-9281.

18. Caldarelli G, Castellano C, Petri A. Criticality in models for fracture in disordered media. Physica A: Statistical Mechanics and its Applications 1999; 270(1):15-20.

19. Parisi A, Caldarelli G. Self-affine properties of fractures in brittle materials. Physica A: Statistical Mechanics and its Applications 2000; 280(1):161-165.

20. Ostoja-Starzewski M, Sheng PY, Alzebdeh K. Spring network models in elasticity and fracture of composites and polycrystals. Computational Materials Science 1996; 7(1-2):82-93.

21. Monette L, Anderson MP. Elastic and fracture properties of the two-dimensional triangular and square lattices. Modelling and Simulation in Materials Science and Engineering 1994; 2(1):53-66.

22. Cundall PA, Strack ODL. A discrete numerical model for granular assemblies. Geotechnique 1979; 29(1):47-65.

23. Rougier E, Munjiza A, John NWM. Numerical comparison of some explicit time integration schemes used in DEM, FEM/DEM and molecular dynamics. International Journal for Numerical Methods in Engineering 2004; 61:856-879.

24. Otter JRH, Cassell AC, Hobbs RE. Dynamic relaxation (Paper no. 6986). Proceedings-Institution of Civil Engineers 1966; 35:633-656. 
25. Li QM, Lu YB, Meng H. Further investigation on the dynamic compressive strength enhancement of concrete-like materials based on split Hopkinson pressure bar tests. Part II: numerical simulations. International Journal of Impact Engineering 2009; 36:1335-1345.

26. Zhang ZN, Ge XR. Micromechanical consideration of tensile crack behavior based on virtual internal bond in contrast to cohesive stress. Theoretical and Applied Fracture Mechanics 2005; 43(3):342-359.

27. Zhao GF, A microstructure based model for rock materials. Proceedings of 1st EPFL Doctoral Conference in Mechanics, Advances in Modern Aspects of Mechanics, 2010; 37-40.

28. Tadmor EB, Ortiz M, Phillips R. Quasicontinuum analysis of defects in solids. Philosophical Magazine A 1996; 73(6):1529-1593.

29. Marsden JE, Hughes TJR. Mathematical Foundations of Elasticity. Prentice-Hall: Englewood Cliffs, NJ, 1983.

30. Potyondy DO, Cundall PA. A bonded-particle model for rock. International Journal of Rock Mechanics and Mining Science 2004; 41:1329-1364.

31. Lakes RS, Lee T, Bersie A, Wang YC. Extreme damping in composite materials with negative-stiffness inclusions. Nature 2001; 410:565-567.

32. Griffiths DV, Mustoe GGW. Modelling of elastic continua using a grillage of structural elements based on discrete element concepts. International Journal for Numerical Methods in Engineering 2001; 50:1759-1775.

33. Chen SG, Zhao J. A study of UDEC modelling for blast wave propagation in jointed rock masses. International Journal of Rock Mechanics and Mining 1998; 35(1):93-99.

34. Zhu WC, Tang CA. Numerical simulation of Brazilian disk rock failure under static and dynamic loading. International Journal of Rock Mechanics and Mining Sciences 2006; 43(2):236-252.

35. Rinehart JS, Pearson J. Behavior of Metals Under Impulsive Loads. Dover: New York, 1965.

36. Brara A, Camborde F, Klepaczko JR, Mariotti C. Experimental and numerical study of concrete at high strain rates in tension. Mechanics of Materials 2001; 33:33-45. 